\title{
Light Generation in Lead Halide Perovskite Nanocrystals: LEDs, Color Converters, Lasers, and Other Applications
}

\author{
Fei Yan, Swee Tiam Tan, Xiao Li,* and Hilmi Volkan Demir*
}

Facile solution processing lead halide perovskite nanocrystals (LHP-NCs) exhibit superior properties in light generation, including a wide color gamut, a high flexibility for tuning emissive wavelengths, a great defect tolerance and resulting high quantum yield; and intriguing electric feature of ambipolar transport with moderate and comparable mobility. As a result, LHP-NCs have accomplished great achievements in various light generation applications, including color converters for lighting and display, light-emitting diodes, low threshold lasing, X-ray scintillators, and single photon emitters. Herein, the considerable progress that has been made thus far is reviewed along with the current challenges and future prospects in the light generation applications of LHP-NCs.

\section{Introduction}

Light emission from lead halide perovskites (LHPs) was reported over a decade ago; however, weak emissions at room temperature hindered the application of LHPs in light-emitting devices because LHPs did not show any electroluminescence at the time..$^{[1-3]}$ In the past few years, LHPs have returned to the spotlight in the form of nanoscale emitters because of

Dr. F. Yan, Dr. S. T. Tan, Prof. H. V. Demir

LUMINOUS! Center of Excellence for Semiconductor Lighting and Displays

TPI-The Photonics Institute

School of Electrical and Electronic Engineering

Nanyang Technological University

Singapore 639798, Singapore

E-mail: HVDEMIR@ntu.edu.sg

Prof. X. Li

School of Chemical Engineering

University of Science and Technology Liaoning

Anshan 114051, P. R. China

E-mail: lixiao@ustl.edu.cn

H. V. Demir

Division of Physics and Applied Physics

School of Physical and Mathematical Sciences

Nanyang Technological University

Singapore 639798, Singapore

H. V. Demir

Department of Electrical and Electronics Engineering

Department of Physics

UNAM-Institute of Materials Science and Nanotechnology

Bilkent University

Ankara 06800, Turkey

The ORCID identification number(s) for the author(s) of this article can be found under https://doi.org/10.1002/smll.201902079.

DOI: 10.1002/smll.201902079 their superior features in light generation and resulting applications. ${ }^{[4-21]}$ Low-cost solution processing techniques are used to fabricate LHP nanocrystals (LHP-NCs), and impressive achievements have been made in using LHP-NCs in high-brightness light-emitting diodes (LEDs) ${ }^{[4-8]}$ with high external quantum efficiency (EQE) and as color converters for lighting and full-color displays that have a wide color gamut and near-unity luminous efficiency. ${ }^{[9-11]}$ The prompt emission of LHPNCs has resulted in their being selected as color converters for light indoor fidelity (Li-Fi) in short-distance wireless data transmission. ${ }^{[12,13]}$ As the most attractive application, red and green LEDs exhibit much higher performances with EQEs $>20 \%$ and high brightness that are comparable to organic LEDs and CdSe quantum dot LEDs; ${ }^{[4-7]}$ however, blue LEDs still have an EQE of $\approx 5.7 \%$ and a maximum brightness of $3780 \mathrm{~cd} \mathrm{~m}^{-2} \cdot{ }^{88}$ LHP-NCs have narrow band emissions and are therefore not the best color convertors for white LEDs in lighting; however, low-cost phosphors exhibit similar performance to lanthanide based white LEDs. ${ }^{[9-11]}$ In addition to applications based on efficient luminescence under low excitation density, LHP-NCs exhibit optically pumped low-threshold lasing and proof-of-principle electrically excited lasing. ${ }^{[14-17]}$ Nonclassic applications exploiting LHP-NCs have been extended to the direct detection of ionizing radiation, e.g., $\mathrm{X}$-rays and $\gamma$-rays in which high-energy X-ray photons are converted to bright visible light and to single photon emitters that can be used in quantum technologies for communication, metrology, and encryption. ${ }^{[18-21]}$

Generally, LHP-NCs can be represented in the form $\mathrm{ABX}_{3}$, where site- $\mathrm{A}$ is an amine or cesium, site- $\mathrm{B}$ is lead, and site- $\mathrm{X}$ is a halogen. ${ }^{[22-24]}$ By varying the components, LHP-NCs can exhibit independent properties with small deviations, including a high charge carrier mobility, ${ }^{[25,26]}$ excellent defect tolerance, ${ }^{[27,28]}$ efficient luminescence, ${ }^{[29,30]}$ and narrow emissive spectra ${ }^{[22-30]}$ that cover significantly different wavelengths from the near-ultraviolet to the near-infrared. ${ }^{[22-24]}$ As the use of LHP-NCs in various applications has grown rapidly, environmental concerns about the use of toxic lead have emerged. Recently, impressive progress has been made using direct-band lead-free double halide perovskites as efficient emitters. ${ }^{[9]}$ Most LHP-NCs can be synthesized through a facile solution reaction, e.g., ligand-assisted reprecipitation in an ambient environment and hot injection in a nitrogen atmosphere, followed by dispersion in a low dielectric constant solvent, e.g., toluene, to form a homogeneous colloidal solution. ${ }^{[31-39]}$ In situ antisolvent 
processing is another technique that can be used to fabricate high-quality LHP-NCs, mostly in the form of solid thin films, using precursor solutions. ${ }^{[40-43]}$

First, a rough introduction of the fundamental properties of LHP-NCs is presented for a better understanding. We are well aware of the important role that the synthesis of LHPNCs plays in determining their characteristics; however, we do not focus on synthesis in this Review. Instead we summarize the latest progress in research on the light generation capabilities of LHP-NCs and the resulting applications, including LEDs, color converters, lasing, nonclassical X-ray scintillators, and single photon emitters; insights into the development, challenges, and prospects are also discussed. We hope this Review provides inspiration for subsequent studies or new potential applications based on LHP-NCs and other materials.

\section{Fundamental Properties of LHP-NCs}

\subsection{Composition and Structure}

In practice, the defects of vacancies or surface dangling bonds and grain boundaries make it difficult to represent LHP-NCs by a stoichiometric formula. When grain boundaries are neglected, under ideal conditions, LHP-NCs can be expressed by the chemical formula $(R)_{2}(\mathrm{~A})_{n-1} \mathrm{~B}_{n} \mathrm{X}_{3 n+1}$, where site-A is an amine cation, e.g., formamidinium (FA) or methylammonium (MA) or $\mathrm{Cs}^{+}$; site- $\mathrm{B}$ is $\mathrm{Pb}^{2+}$; and site- $\mathrm{X}$ is $\mathrm{Cl}^{-}$, $\mathrm{Br}^{-}$, or $\mathrm{I}^{-}{ }^{-[29,30,35,36,44]}$ For $n=1$, the abovementioned formula can be predigested into a monolayer of $2 \mathrm{D} R_{2} \mathrm{BX}_{4}$, where $R$ is a molecular ligand sandwiching a corner-sharing $\left[\mathrm{PbX}_{6}\right]^{4-}$ octahedral perovskite sheet (Figure 1a). ${ }^{[30,35]}$ In extreme cases, when $n=\infty$, the abovementioned formula can be approximated by $\mathrm{ABX}_{3}$, which corresponds to 3D LHP-NCs (Figure 1a). ${ }^{[30,36]}$ In both 2D and 3D LHP-NCs, the corner-sharing $\left[\mathrm{PbX}_{6}\right]^{4-}$ octahedron is the core fragment, and $\mathrm{Pb}^{2+}$ always occupies the pivot site of an octahedron formed by halogen anions. This site also corresponds to the center of the cube formed by A-cations in 3D LHPs. ${ }^{[22-24]}$

In lead-free halide perovskites, the $\mathrm{Pb}^{+2}$ in site- $\mathrm{B}$ is equivalently substituted by a monovalent metal cation (such as $\mathrm{Cu}^{+}, \mathrm{Ag}^{+}$, and $\mathrm{Na}^{+}$) and a trivalent metal cation (such as $\mathrm{Bi}^{3+}, \mathrm{In}^{3+}$, and $\mathrm{Sb}^{3+}$ ); that is, the formula becomes $\mathrm{A}_{2} \mathrm{BB}^{\prime} \mathrm{X}_{6}$ (Figure 1b). ${ }^{[45,46]}$ Only one trivalent metal cation $\left(\mathrm{Bi}^{3+}\right.$ or $\left.\mathrm{Sb}^{3+}\right)$ can occupy site- $B$, thus producing another type of halide double perovskite with the formula $\mathrm{A}_{3} \mathrm{~B}_{2} \mathrm{X}_{9}$, where site- $\mathrm{A}$ and site- $\mathrm{X}$ are the same as in classical $\mathrm{APbX}_{3}$ LHPs. ${ }^{[47]}$

\subsection{Energy Diagram and Defect Tolerance}

The energy level configuration of LHPs is mainly determined by the site- $B$ and site- $X$ components. ${ }^{22-24,27,28,48,49]}$ The valence band for LHPs is hybridized by the $n p$ orbitals of the halogens and the $6 \mathrm{~s}$ orbitals of lead, whereas the conductive band is mainly determined by the lead $6 \mathrm{p}$ orbitals with a minor contribution from the $\mathrm{np}$ orbitals of the halogens (Figure 1c). ${ }^{[48,49]}$ Thus, the bandgap of LHPs can be widely tuned by exchanging halogen species with different np orbital

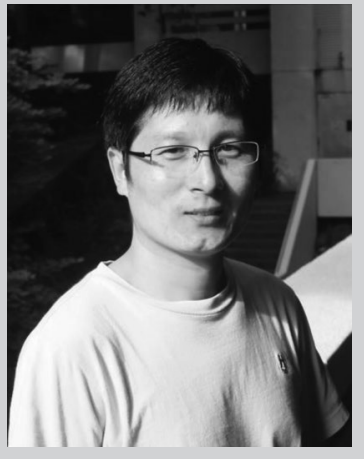

Fei Yan received his Ph.D. degree from Changchun Institute of Optics, Fine Mechanics and Physics, Chinese Academy of Sciences, P. R. China, in 2010. He is currently a postdoctoral research fellow of LUMINOUS! Center of Excellence for Semiconductor Lighting and Displays Nanyang Technological University, Singapore. His current research interests include soft material (organic molecules and halide perovskites nanocrystals) light-emitting devices and physics.

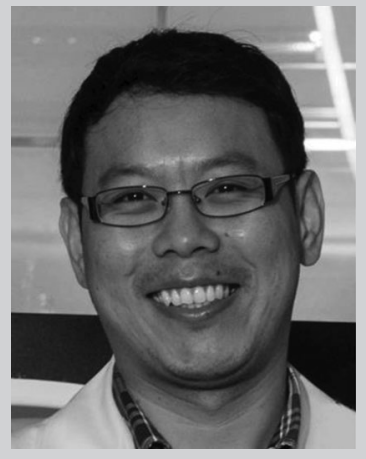

Swee Tiam Tan received his B. Eng. and Ph.D. degrees from Nanyang Technological University, Singapore, in 2003 and 2007, respectively. From 2007 to 2010 , he was with the Institute of Microelectroncis, A*STAR, Singapore, where he led the work on wide-bandgap materials. Since 2010, he has been with the Nanyang Technological University,

Singapore, where he is currently the Programme Manager of LUMINOUS! Center of Excellence.

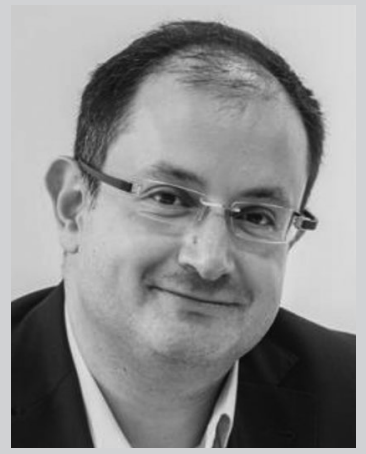

Hilmi Volkan Demir received B.S. (1998) from Bilkent University, and M.S. (2000) and Ph.D. (2004) from Stanford University. As Singapore's NRF Fellow, he is currently a professor of electrical engineering, physics, and materials at NTU and Director of LUMINOUS! Center of Excellence for Semiconductor Lighting and Displays. Concurrently, he holds appointment at Bilkent University (his alma mater) and National Nanotechnology Research Center (UNAM). His current research interests include semiconductor nanocrystals and perovskite optoelectronics.

energy levels (Cl-3p, Br-4p, and I-5p). ${ }^{[48,49]}$ Changing the halogen species from chlorine to iodine drastically shifts the light emitted from the resulting LHPs from near-ultraviolet to near-infrared (Figure 1d). ${ }^{[22-24,31]}$ Exploiting alloyed halide species at various ratios to occupy site- $\mathrm{X}$ allows the flexible tuning of the bandgap of the resulting LHPs, resulting in 

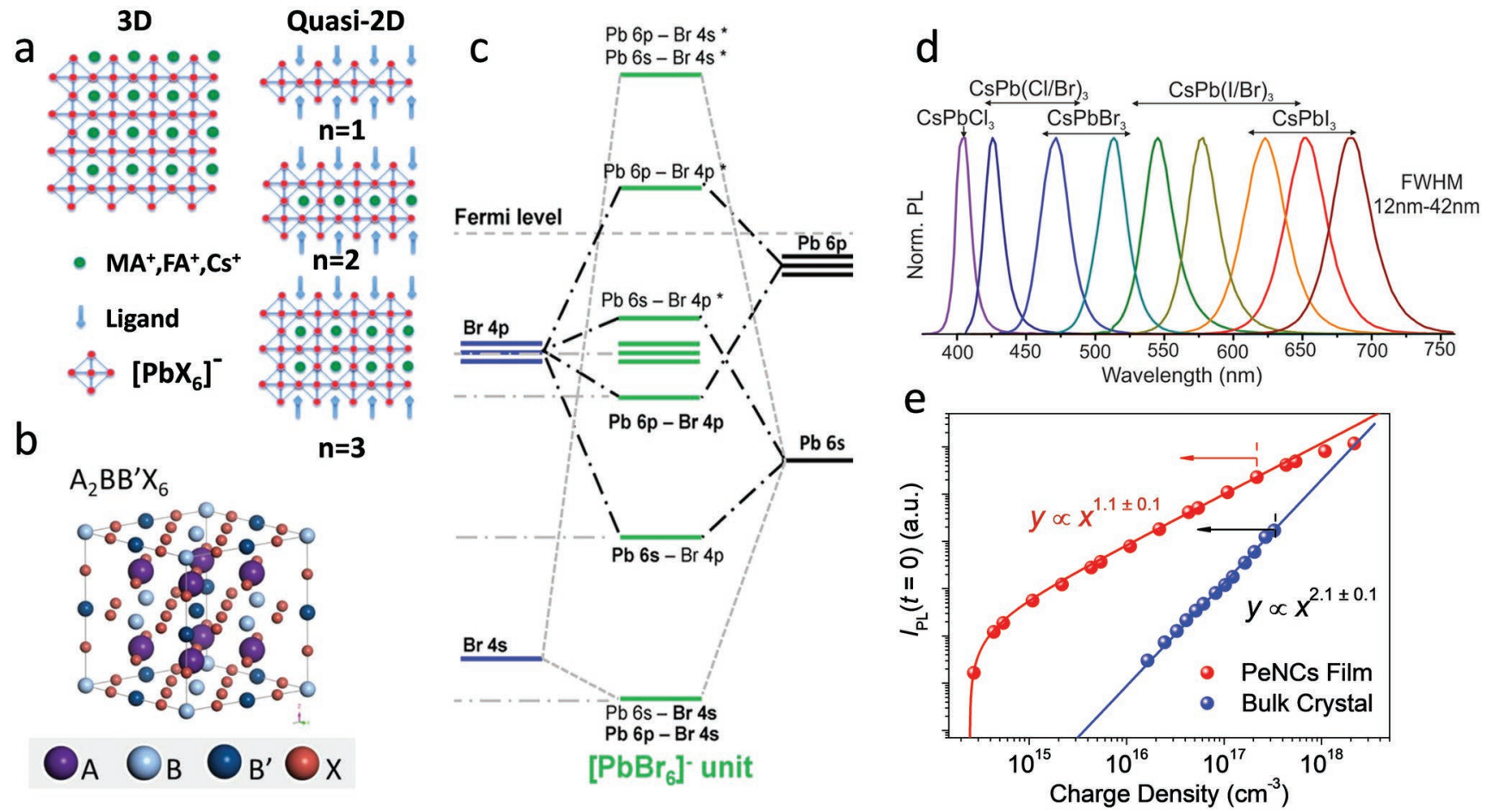

Figure 1. a) Schematic structures of the 3D and quasi-2D LHP-NCs. Reproduced with permission. ${ }^{[30]}$ Copyright 2016, Springer Nature. b) Schematic of the $\mathrm{A}_{2} \mathrm{BB}^{\prime} \mathrm{X}_{6}$ perovskite structure. Reproduced with permission. ${ }^{[45]}$ Copyright 2018, American Chemical Society. c) Schematic energy diagrams of $\mathrm{MAPbBr}_{3}$. Other LHPs, such as $\mathrm{MAPbl}_{3}$ and $\mathrm{CsPbBr}_{3}$ indicate a similar band characteristics. ${ }^{[27]}$ Reproduced with permission. ${ }^{[48]}$ Copyright 2017, American Chemical Society. d) The migration of PL spectra of $\mathrm{CsPbX}_{3} \mathrm{NCs}\left(\mathrm{X}=\mathrm{Cl}, \mathrm{Br}\right.$, I). Reproduced with permission. ${ }^{[31]}$ Copyright 2015, American Chemical Society. e) The dependence of the initial time PL intensity $\left(I_{\mathrm{PL}}(t=0)\right)$ on photon-injected charge carrier density $($ excitation at $400 \mathrm{~nm}, 1 \mathrm{KHz}$, $50 \mathrm{fs}$ ). A bimolecular recombination in bulky $\mathrm{MAPbBr}_{3}$ can be confirmed by the quadratic behavior, and the linear dependence suggests an exciton radiation process in $\mathrm{MAPbBr}_{3} \mathrm{NCs}$. Reproduced with permission. ${ }^{[59]}$ Copyright 2018, American Chemical Society.

continuously adjustable emissive spectra across the entire visible range (Figure 1d). ${ }^{[31,50-54]}$ By comparison, the A-cation plays a minor role in determining the energy level configuration of LHPs. Changing the A-cation from $\mathrm{Cs}^{+}$to $\mathrm{MA}^{+}$ modulates the emissive spectrum of $\mathrm{APbBr}_{3}$ by a smaller redshift. ${ }^{[5]}$

Unlike classic semiconductors with bandgap defined by the difference between bonding valence band maxima (VBM) and antibonding conduction band minima (CBM), the VBM of LHPs is formed by an antibonding interaction of X-np orbital and $\mathrm{Pb}-6 \mathrm{~s}$ orbital, and the nonbonding CBM is mainly decided by the $\mathrm{Pb}-6 \mathrm{p}$ orbitals along with a slight contribution from X-np orbitals. ${ }^{[27,28,48,56-58]}$ Thus, the absence of bonding-antibonding interaction between these special CB and VB results in a high defect tolerance, where the highest defect states, primarily A-cation and $\mathrm{X}$-anion vacancies, lie within the valence or conduction bands instead of in the bandgap (Figure 1c). ${ }^{[27,28,48,56-58]}$ The potential deep trap states formed by interstitial and antisite defects can be neglected because of their high formation energy. A clean bandgap structure enables LHP-NCs to produce an energy-efficient and narrow band emission, even in the absence of ligands for passivating surface defects. ${ }^{[27,28,56-58]}$ Any remaining shallow bandgap states caused by surface defects can be effectively passivated using ligands. ${ }^{[4-8,27,28]}$ The unique energy diagrams and the resulting high defect tolerance of LHP-NCs result in superior optical and electrical characteristics even when
LHP-NCs are fabricated by a crude synthesis However, the low luminous efficiency of LHP-NCs at low excitation densities suggests that there is still considerable loss from trap-induced nonradiative recombination. ${ }^{[4-7,22,59]}$ Hence, the most efficient LHP-NCs are crystalline and surrounded by passivating ligands. ${ }^{4-7,14-22,59]}$

\subsection{Exciton Binding Energy and Recombination}

Binding energies of only dozens of $\mathrm{meV}^{[60-66]}$ indicate that the dissociation of formed Wannier-Mott excitons ${ }^{[60-63]}$ into free charge carriers dominates radiative recombination; this behavior is consistent with the low overlap of electron/hole wave functions. ${ }^{[67]}$ Luminescence of bulky LHPs is generated from delocalized electrons and holes by a second-order bimolecular recombination at a slow rate (Figure 1e). ${ }^{[59,64,67-69]}$ Furthermore, due to a strong spin-orbit coupling caused by the presence of lead, a heavy atom, and inversion symmetry breaking, the resulting Rashba effect results in a slight deviation of the direct band structure, in agreement with the observed indirect tail states in LHPs; thus, the radiative recombination would be limited. ${ }^{[66,70]}$ Consequently, the photoluminescence quantum yield (PLQY) of bulky LHPs is approximately $10-20 \%$ at a moderate excitation density level, ${ }^{[59,69]}$ which is consistent with the large equivalent parallel resistance of bulky LHP solar cells. The recombination rate can be increased by enhancing the overlap between the hole and electron wave 

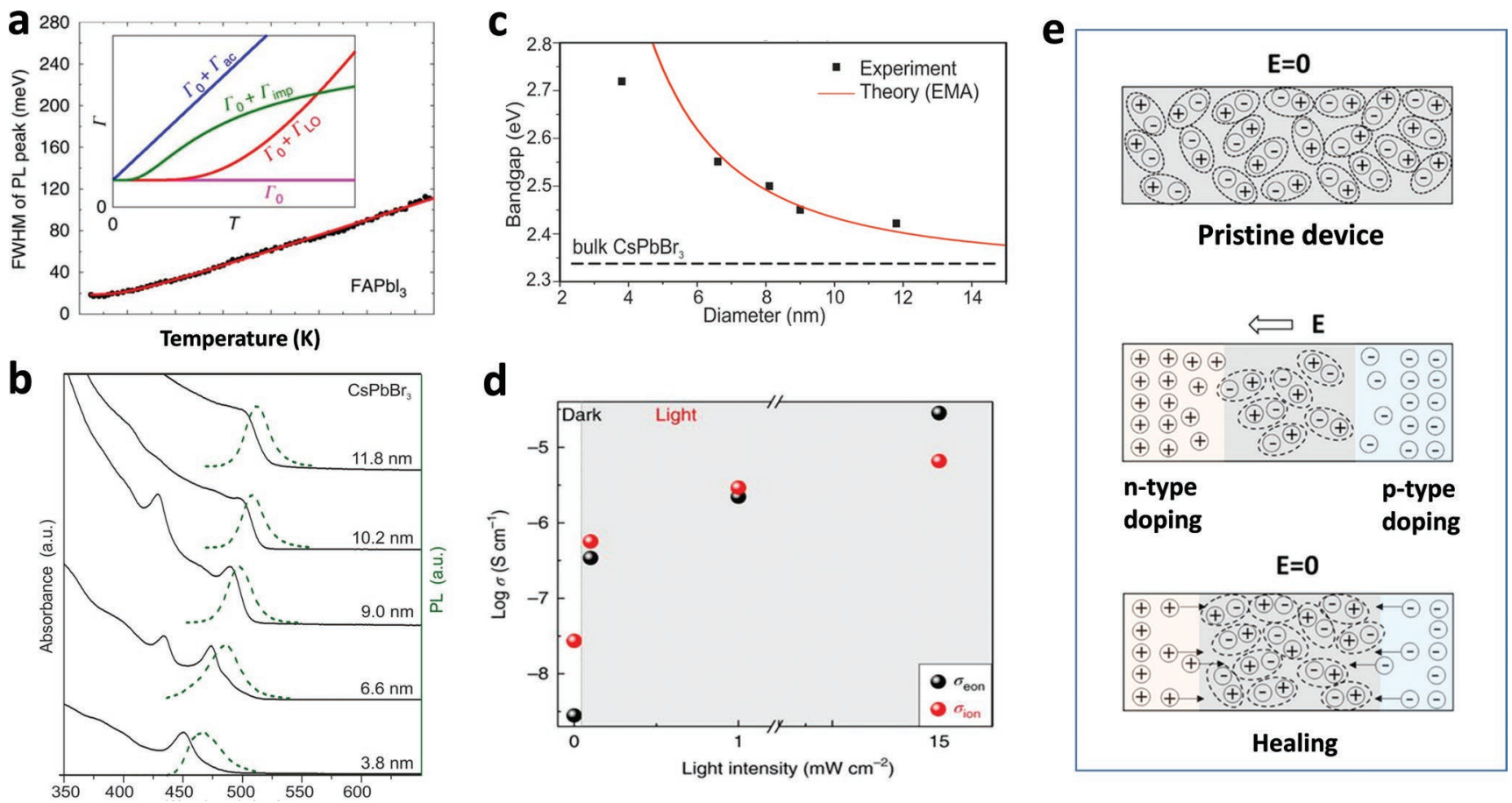

Figure 2. a) The dependence of PL linewidth on temperature for $\mathrm{FAPb}_{3}$ thin films. The solid red lines are fits of $\Gamma(T)=\Gamma_{0}+\Gamma_{\mathrm{LO}}$. The inset shows the functional form of the temperature dependence of the contributions to PL linewidth from inhomogeneous broadening $\left(\Gamma_{0}\right)$, Fröhlich coupling between charge carriers and LO phonons $\left(\Gamma_{\mathrm{LO}}\right)$ and acoustic phonons $\left(\Gamma_{\mathrm{ac}}\right)$, and scattering from ionized impurities $\left(\Gamma_{\text {imp }}\right)$. Reproduced with permission. ${ }^{[79]}$ Copyright 2016, Springer Nature. b) Migration of absorption and PL spectra with a decreasing $\mathrm{CsPbBr}_{3} \mathrm{NCs}$ size in the range of 5-12 nm. Within the effective mass approximation (EMA), the effective Bohr diameters of Wannier-Mott excitons for $\mathrm{CsPbBr}_{3}$ are around $7 \mathrm{~nm}$. Reproduced with permission. ${ }^{[3]}$ Copyright 2015, American Chemical Society. c) The dependence of bandgap on the size of $\mathrm{CsPbBr}_{3}$ NCs. Reproduced with permission. ${ }^{[3]}$ Copyright 2015, American Chemical Society. d) Conductivity of electron and ion extracted from d.c. galvanostatic polarization testing. Both ionic and electronic can be boosted under light. Reproduced with permission. ${ }^{[52]}$ Copyright 2018, Springer Nature. e) Schematic ion migration of LHPs. Uniform distributed ions in a pristine LHP film take a directional motion under an electrical field, leading to a p-type doping near the LHPs/anode interface and n-type doping near the LHP/cathode interface. After the electric field is removed, ions diffuse back for healing LHPs. Reproduced with permission. ${ }^{[124]}$ Copyright 2017, American Chemical Society.

functions via spatial confinement by small domains of LHPNCs with dimensions of several nanometers, ${ }^{[1,72]}$ especially atomically thin quasi-2D LHP-NCs that are nanoplatelets (NPLs) containing a few $\left[\mathrm{PbBr}_{6}\right]^{4-}$ monolayers. The remarkable exciton dissociation presented in bulky LHPs is suppressed effectively in LHP-NCs because of their exciton binding energy up to hundreds of $m e V,{ }^{[73-77]}$ and their fast first-order exciton recombination regime overwhelms the slow bimolecular recombination in light generation (Figure 1e). ${ }^{[59,69]}$

As a result of electron-hole exchanging, the exciton of $\mathrm{CsPbBr}$ NCs splits into a singlet state and a threefold degenerate triplet states. It is proposed that a strong Rashba effect lifts the degeneracy of the triplet state and alters the order of all sublevels, making a bright triplet state the lowest one. With this special characteristic differing from classical semiconductors, $\mathrm{CsPbBr}_{3}$ NCs indicate highly efficient luminescence. ${ }^{[78]}$ This mechanism of bright triplet exciton could be same to other LHP-NC species in general because of their similar component, structure, and performance achieved, though experimental evidence is still absent as yet.

\subsection{Optical Features}

The polar $\mathrm{Pb}-\mathrm{X}$ bond in LHP-NCs enables the Fröhlich coupling of charge carriers to longitudinal optical phonons, which plays a predominant role in determining a narrow linewidth of the emissive spectra at room temperature (Figure 2a). ${ }^{[60,79,80]}$ By contrast, there is negligible linewidth broadening from other intrinsic factors, e.g., the interaction between a charge carrier and an acoustic phonon, and extrinsic factors, e.g., defects and impurities (Figure 2a). [27,28,58,60,79]

Because of the small exciton Bohr radius, even with a confinement of small grains with dimensions of several nanometers, the emissive spectra for LHP-NCs have a slight blueshift as the size decreases and are almost the same as those of bulky LHP emitters (Figure 2b). ${ }^{[4-6,81,82]}$ A further reduction of the dimensions of LHP-NCs to the level of their exciton Bohr radius, ${ }^{[31]}$ e.g., monolayer quasi-2D LHP-NCs, can produce a significant quantum confinement effect and an associated higher bandgap and a blueshift in the emissive spectrum of over $200 \mathrm{meV}$ (Figure 2b,c). ${ }^{[31,35,75-77]}$ Moreover, the overlap between the absorption and emission bands results in photon recycling inside the LHP-NC film, which enhances the overall outcoupling efficiency by increasing the utilization of photons from the emissive film. ${ }^{[83]}$

\subsection{Charge Carrier Transport}

Among various LHP-NC applications, electrically driven applications are the most attractive, e.g., LEDs; thus, 
Table 1. Summary of LEDs using LHP-NCs as emitters.

\begin{tabular}{|c|c|c|c|c|c|}
\hline Emitter & Device structure & $\lambda[\mathrm{nm}]$ & $\mathrm{EQE}_{\max }[\%]$ & $L_{\max }\left[\mathrm{cd} \mathrm{m}^{-2}\right]$ & Refs. \\
\hline $\mathrm{CsPb}_{3} \mathrm{NCs}$ & $\mathrm{Au} / \mathrm{MoO}_{3} / \mathrm{TCTA} /$ emitter/PEI/ZnO/Al & 692 & 8.2 & 827 & {$[95]$} \\
\hline $\mathrm{MAPbBr}_{3}$ QDs & ITO/PEDOT:PSS/PVK/emitter/TPBi/LiF/Al & 520 & 1.64 & 1635 & [97] \\
\hline$(\mathrm{PEA})_{2} \mathrm{MA}_{m-1} \mathrm{~Pb}_{m} \mathrm{Br}_{m+1} \mathrm{NCs}$ & FTO/Buf-HIL/emitter/TPBi/LiF/Al & 520 & 4.98 & 2935 & {$[98]$} \\
\hline BAX:MAPbX ${ }_{3}$ & TO/HTL/emitter/TPBi/LiF/Al & 750 & 10.4 & - & [99] \\
\hline $\mathrm{PEA}_{2}\left(\mathrm{FAPbBr}_{3}\right)_{n-1} \mathrm{PbBr}_{4} \mathrm{NPLS}$ & ITO/PEDOT:PSS/emitter/TPBi/LiF/Al & 532 & 14.3 & 7000 & {$[96]$} \\
\hline $\mathrm{FAPbBr}_{3} \mathrm{NCs}$ & ITO/PEDOT:PSS/emitter/B3PYMPM/Cs $\mathrm{CO}_{3} / \mathrm{Al}$ & 530 & & 2714 & [101] \\
\hline $\mathrm{CsPbBr}_{3} \mathrm{NCs}$ & ITO/PEDOT:PSS/emitter/B3PYMPM/Ca/Al & 522 & 1.75 & 2721 & [110] \\
\hline $\mathrm{CsPbBr}_{3} / \mathrm{MABr}$ quasi-core/shell & ITO/PEDOT:PSS/emitter/B3PYMPM/LiF/Al & 531 & 20.3 & 14000 & [4] \\
\hline quasi-3D $\left.(\mathrm{NMA})_{2}(\mathrm{FA})_{m-1} \mathrm{~Pb}_{m}\right|_{3 m+1}$ & ITO/MZO/PEIE/emitter/TFB:PFO/MoO $/ \mathrm{Au}$ & 795 & 20.1 & - & [6] \\
\hline $\mathrm{CsPbBr}_{3} \mathrm{QDs}_{-} \mathrm{ZnBr}_{2}$ & ITO/PEDOT:PSS/PTTA/emitter/QDS/TPBi/LiF/AI & 520 & 16.48 & 76940 & {$[5]$} \\
\hline $\mathrm{CsPb}(\mathrm{Br} / \mathrm{I})_{3} \mathrm{QDs}$ & ITO/PEDOT:PSS/poly-TPD/emitter/NPD / $\mathrm{MoO}_{3} / \mathrm{Al}$ & - & 21.3 & & {$[7]$} \\
\hline $\mathrm{MAPbBr}_{3} \mathrm{NCs}$ & ITO/PEDOT:PSS/emitter/B3PYMPM:TPBi/ $\mathrm{Cs}_{2} \mathrm{CO}_{3} / \mathrm{Al}$ & 524 & 12.9 & 43000 & [59] \\
\hline $\mathrm{CsPb}(\mathrm{Br} / \mathrm{Cl})_{3} \mathrm{NCs}$ & ITO/PEDOT:PSS/poly-TPD/CBP/emitter/B3PYMPM/LiF/AI & 463 & 1.2 & 318 & [102] \\
\hline $\mathrm{CsPbBr}_{3} \mathrm{NCs}$ & ITO/PEDOT:PSS/poly-TPD/CBP/emitter/TPBi/LiF/Al & 513 & 9.7 & 2269 & [105] \\
\hline$(\mathrm{PEA})_{2} \mathrm{PbBr}_{4} \mathrm{NPLs}$ & ITO/PEDOT:PSS/PVK/TAPC/emitter/TPBi/Ca/Ag & - & 0.3 & 147 & [106] \\
\hline $\mathrm{MAPbl}_{3} \mathrm{NCs}$ & ITO/PEDOT:PSS/poly-TPD/emitter/ LiF/Al & 635 & 2.75 & - & [107] \\
\hline $\mathrm{CsPbBr}_{3} \mathrm{NCs}$ & ITO/PEDOT:PSS/poly-TPD/emitter/ $\mathrm{Cs}_{2} \mathrm{CO}_{3} / \mathrm{Al}$ & 522 & 1.7 & 1562 & [109] \\
\hline
\end{tabular}

charge-carrier transport is a critical characteristic for LHP-NC films. Charge carrier transport in LHP-NCs is a complex process, and several intrinsic and extrinsic factors determine and modulate charge carrier mobility. The mechanically soft $\left[\mathrm{PbX}_{3}\right]^{-}$sublattice results in charge carrier transport as large polarons with radii of $40-50 \AA$ in $\mathrm{MAPbI}_{3},{ }^{[84]}$ exceeding the lattice constant; in theory, a high charge carrier mobility up to thousands of $\mathrm{cm}^{2} \mathrm{~V}^{-1} \mathrm{~s}^{-1}$ can be produced by screening charge carriers from a scattering effect by defects, other charge carriers, and phonons, ${ }^{[84-87]}$ which is consistent with the small effective masses of electrons and holes. ${ }^{[88-90]}$ As in the linewidth determination of the emissive spectrum, the interaction between charge carriers and longitudinal optical phonons follows the Fröhlich model and plays a critical role in charge transport, where the upper limit of the charge carrier mobility is set at $\approx 100 \mathrm{~cm}^{2} \mathrm{~V}^{-1} \mathrm{~s}^{-1}$, in agreement with experimental results. ${ }^{[25,84,91]}$ However, the Fröhlich model prediction of the temperature dependence of the charge carrier mobility deviates from the experimental results of $\mu \propto T^{-1.5}$ at room temperature, ${ }^{[25,79,84,86,91]}$ suggesting that the charge carrier mobility needs to be determined more carefully. Consistent with this high mobility, the excellent defect tolerance of the clean bandgap points toward a low level of trap scattering for charge carrier transport.

Another advantage offered by LHP-NCs is natural ambipolar charge carrier transport, which is comparable to hole and electron mobilities and is essential for high energy-efficiency in electrically driven LEDs. ${ }^{[25,59,89,92]}$ Apart from the intrinsic factors mentioned above, both the hole and electron mobilities of a LHP-NC film remain at a modest level of $\approx 0.5 \mathrm{~cm}^{2} \mathrm{~V}^{-1} \mathrm{~s}^{-1}$,[59] even with extrinsic scattering from grain boundaries, impurities, and surrounding ligands. In addition to a high hole/electron mobility, the migration of the A-cation and halide anions contribute to the superior charge carrier transport capability of
LHP-NCs (Figure 2d), ${ }^{[52]}$ especially in LEDs that are driven by high external electrical fields. The contribution of ion migration to current conduction makes it difficult to extract the true hole/ electron mobility from complex experimental results obtained from space charge limited current, time-of-flight, hall-mobility measurements, etc. ${ }^{[93]}$ Directional ion migration under an electrical field degrades LHP-NCs and changes their lattice structure, which makes the hole/electron transport mechanism more complex. ${ }^{[51,52,93]}$

\section{LEDs Exploiting LHP-NC Emitters}

LEDs using LHP-NCs as emitters exhibit superior luminescence and charge carrier transport and have attracted the most attention for various applications. (Table 1). ${ }^{[4-7,59,94-111]}$ Electrical driving almost does not change the superior optical features of LHP-NCs as emitters in the resulting LEDs, including their high color purity, a wide color gamut, and a stable spectrum that is independent of the applied driving voltage. ${ }^{[4-7,59,94-111]}$ Under electrical excitation, LHP-NCs consisting of mixed halides would undergo spectral splitting; however, RGB primary color LEDs that use single-halide LHP-NC emitters still exhibit a wide color gamut that can display most natural colors in resulting full color displays.

\subsection{Device Structure and Fabrication}

Usually, a conductive poly(3,4-ethylenedioxythiophene)poly(styrenesulfonate) (PEDOT:PSS) film is selected as a modification layer of the indium tin oxide (ITO) electrode for improving hole injection. ${ }^{[4,5,7,59,96,97]}$ As a water-soluble material, a PEDOT:PSS film has a high surface energy that facilitates 
subsequent film deposition and good passivity against most organic solvents. ${ }^{[112,113]}$ However, these metallic polymer films were supposed to be exciton quenchers, ${ }^{[114-116]}$ therefore, a polymer with a low charge carrier density, e.g., poly(4-butylphenyldiphenylamine) (poly-TPD), with a matched energy level and charge carrier mobility is selected as a buffer layer for eliminating the quenching at the interface. ${ }^{[5,7,96,97,99]}$ Meanwhile, as an intrinsic hole transport layer, the high lying of the lowest unoccupied molecular orbital levels can block the overflow of electrons, especially at a high driving current density level. ${ }^{[5,7,105]}$ In principle, the low hole mobility of an organic molecular layer between PEDOT:PSS and LHP-NCs can help suppress excess hole injection into recombination zone..$^{[5,7,105]}$ Interestingly, the LHP-NC LEDs with bare PEDOT:PSS electrodes also exhibit a high EQE that is over 20\%. ${ }^{[4]}$ In comparison to PEDOT:PSS, some classical hole transport molecule films play a more significant role in quenching the emission of LHP-NCs despite a lower charge carrier density.

As the hole and electron mobilities in LHP-NCS are comparable, the charge carrier supply capability of the electron transport layer must be comparable to the hole side to guarantee a balanced charge carrier injection into the recombination zone. However, the conductivity of the most popular PEDOT:PSS AI 4083 used in LEDs is up to $1.4 \times 10^{-4} \mathrm{~S} \mathrm{~cm}^{-1}$, $[117,118]$ which is several orders of magnitude higher than that of most organic electron transport layers, which have with low conductivities of $\approx 10^{-10}-10^{-6} \mathrm{~S} \mathrm{~cm}^{-1}$;[119] thus, overall, imbalanced charge carriers with dominant hole injection into the device are to be expected. However, the LHP-NC LEDs still exhibit high EQEs of even 20\%.

At a technical level, the compatibility of a solution-proceeding film deposition is a critical consideration in successful LED fabrication. This compatibility mainly requires a high surface energy for the substrate relative to the solution for the deposition of the subsequent film and good passivity against all of the subsequent operations. Charging using oxygen plasma treatment can improve the wetting of the deposited film to the solution for subsequent film deposition. Being ionic crystals, LHP-NCs are sensitive to high dielectric constant environments; thus, solvents with a high dielectric constant solvents are generally excluded from a device fabrication process.

Essential requirements for inverted structure LHP-NC LEDs are similar to those for normal structure devices: the selection of matched functional layers for balanced charge carrier injection and compatibility in solution processing film deposition. ${ }^{[6,95,100,120]}$ Normally, a zinc oxide nanocrystal film is selected as the electron transport layer in inverted structure of LHP-NC LEDs, ${ }^{[6,95,100,120]}$ and a polymer film, e.g., polyethyleneimine ethoxylated, is used to modify the interface. This design is similar to the PEDOT:PSS/ poly-TPD combination in normal structure devices.

\subsection{Charge Carrier Transport and Ion Conductance}

Comparable electron and hole mobilities help to sustain a balance between opposite charge carriers and to reduce the accumulation of charge carriers in the recombination zone and energy loss resulting from Auger nonradiative recombination. In addition to high hole and electron mobilities, the migration of halide anions and A-cations contributes to the superior charge carrier transport under an applied electrical field, ${ }^{[51,52,93,121-123]}$ where a accumulation at the interface leads to p-type and n-type doped injection layers at the corresponding interfaces that facilitate charge carrier injection into an LHP-NC layer (Figure 2e). ${ }^{[124-126]}$ However, ion migration results in undesirable degradation in LHP-NCs, especially under an electrical field, which leads to short operational stability and potential EQE roll-off. ${ }^{[52,124,127,128]}$ With the illumination of photon energies exceeding the bandgap, ion conductivity can be boosted (Figure 2d);[52,129] however, it is not clear whether the self-absorption inside LHP-NCs can trigger this enhancement. In quasi-2D LHP-NCs, ion migration out-of-plane planes can be suppressed by an inhomogeneous structure of an inorganic $\left[\mathrm{PbX}_{6}\right]^{4-}$ sandwiched by insulating ligands with strong dielectric confinement, which improves the stability of the NCs and resulting devices. ${ }^{[72,75,130,131]}$ However, a side-effect is that the charge carrier mobility and conductivity is also suppressed by one order of magnitude (Figure 3a), ${ }^{[74,132]}$ leading to a high driving voltage in the resulting LEDs. ${ }^{[42]}$

\subsection{Blue and Quasi-2D LHP-NC Emitters}

In the past few years, both green and red LHP-NC LEDs have exhibited high EQEs over 20\%, showing promising prospects for full color display. However, blue LHP-NC LEDs are the final color component for full color displays, the development of which still falls behind green and red LHP-NC LEDs, and most reported studies have focused on sky-blue and not deep blue devices with wavelengths shorter than $460 \mathrm{~nm} \cdot{ }^{[42,133,134]}$ The blue LHP-NC emitters mainly include chloride-based 3D NCs and bromide-based quasi-2D NCs, i.e., nanoplatelets. The chloride-based 3D LHP-NCs generally exhibit low energy efficiency and low stability, especially when electrically driven. ${ }^{[135-137]}$ As mentioned above, the deep lying of the valence band determined by chloride results in a high barrier for hole injection into the emissive layer and a high voltage driven LED compared to green and red LEDs. ${ }^{[135-137]}$

In principle, sufficiently small 3D bromide-based LHP-NCs with strong quantum confinement could also emit high-quality blue light; however, the exciton Bohr radii of these devices are comparable to the dimensions of the $\left[\mathrm{PbBr}_{6}\right]^{4-}$ core, ${ }^{[31]}$ which makes it more difficult to control the size and monodispersity of LHP-NCs. ${ }^{[35,82,138]}$ Alternatively, bromide-based quasi-2D NCs with strong quantum confinement and dielectric confinement are regarded as efficient blue emitters compared to their 3D counterparts. ${ }^{[31,35,42,72,75-77]}$ The bandgap of quasi-2D LHP-NCs can be adjusted by a quantized change in the number of $\left[\mathrm{PbBr}_{6}\right]^{4-}$ cells. ${ }^{[75,79,82]}$ Two monolayer $\mathrm{CsPbBr}_{3}$ NPL designs can emit deep blue light with peak wavelengths of $\approx 440 \mathrm{~nm}$, whereas their $3 \mathrm{D}$ counterparts emit green light (Figure 3b). ${ }^{[35,75,82]}$ Moreover, hydrophobic ligands arranged in a preferred orientation protect the $\left[\mathrm{PbBr}_{6}\right]^{4-}$ cell from ambient moisture and suppress ion migration, resulting in efficient emission with long-term stability. ${ }^{[35,42,72,75,82]}$

With comparable effective electron and hole masses and confined electron and hole wave functions, the energy levels of quasi-2D LHP-NCs can expand symmetrically, ${ }^{[88-90]}$ in comparison to their 3D counterparts with the same components. Hence, the slightly increased bandgap $(\approx 0.3 \mathrm{eV})$ does not 

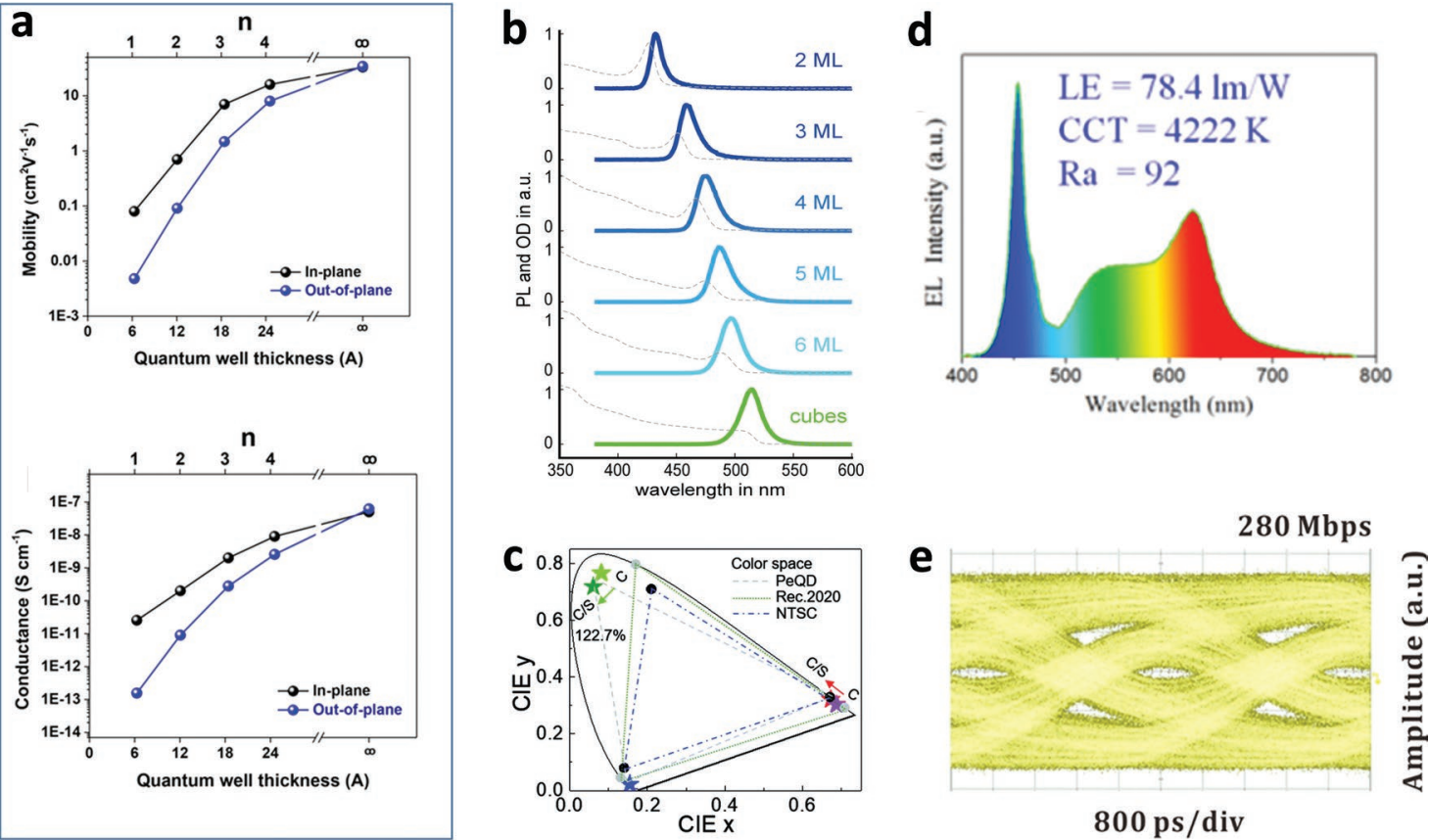

Figure 3. a) Comparison of in-plane and out-of-plane mobility and conductivity with respect to the number of monolayer quasi-2D LHP-NCs. Reproduced with permission. ${ }^{[7]}$ Copyright 2018, American Chemical Society. b) The dependence of PL and absorption spectra on the unit number in colloidal CsPbBr3 NPLs. Reproduced with permission. ${ }^{[75]}$ Copyright 2018, American Chemical Society. c) Comparison of color space of LHP QDs to color coordinate of Rec.2020 and NTSC. Reproduced with permission. ${ }^{[147]}$ Copyright 2018, Royal Society of Chemistry. d) EL spectra of WLED with LHP QD color converter. Reproduced with permission. ${ }^{[154]}$ Copyright 2017, Elsevier. e) Eye diagrams versus data rates of WLEDs ( $\mu$ LED + CsPbBr1.811.2 yellow LHP QDs) at 280 Mbps. Reproduced with permission. ${ }^{[13]}$ Copyright 2018, American Chemical Society.

require as high as a voltage to drive the resulting LEDs. ${ }^{[42,139,140]}$ As in quasi-2D LHP-NCs, insulating ligands arranged in a preferred orientation block the charge carrier transfer out of the $\left[\mathrm{PbX}_{6}\right]^{4-}$ plane (Figure $3 \mathrm{a}$ ), which could be the dominant factor in the high driving voltage. ${ }^{35,42,71,74,82]}$ The current-voltage characteristics of some LEDs using quasi-2D LHP-NC emissive films exhibit a low driving voltage, which may be attributed to the leakage current generated by a rough morphology or pinholes in low-quality emissive films. In particular, the relatively large lateral size of quasi-2D LHP-NCs can result in a chain formation in colloidal solution by vertical stacking, decreasing the luminous efficiency and producing a rough morphology in deposited films and polarized emission. ${ }^{[75,141,142]}$

\subsection{Guidelines for Producing Efficient LHP-NC LEDs}

By definition, a high EQE corresponds to a maximized output of photons with respect to the minimized input of electrons injected into LEDs. Several factors lead to EQE losses in operating LHP-NC LEDs, including the intrinsic characteristics of LHP-NC emitters and extrinsic loss that depend on the device structure. The intrinsic EQE loss in LHP-NCs typically includes two contributions to nonradiative recombination: trap states and the Auger process. ${ }^{[4-7,59,94-111]}$ As a planar device structure limits the low photon outcoupling efficiency to $\approx 20 \%$, the PLQY of the LHP-NC film plays an ultimate role in determining the maximum EQE that resulting LEDs can achieve. ${ }^{[4-7,59]}$ A high defect tolerance does not correspond to the absence of the resulting EQE loss, which is consistent with a low initial EQE at a low driving current density level; ${ }^{[4-7,59,68,94-111]}$ thus, a high-quality LHP-NCs emitter film with a low defect density is required to suppress the EQE loss from trap-mediated nonradiative recombination. Unlike trap-assisted nonradiative recombination, which occurs at all excitation levels, the EQE loss from a multiexciton Auger process only occurs at high excitation levels, which is more applicable to EQE droop at high driving current densities in LEDs and proof-of-principle electrically pumped lasing studies. Moreover, the degradation of LHPNCs driven by an applied field, a high current density and the resulting Joule heating leads to irreversible EQE loss. ${ }^{[51,52,143]}$ Therefore, coating quasi-2D and 3D LHP-NCs to suppress ion migration can reduce EQE loss. ${ }^{[6]}$

The extrinsic factors resulting in EQE loss are related to the device structure and include the leakage current and imbalanced charge carriers. ${ }^{[4,59]}$ In an LED with a low-quality LHP-NC film and charge carrier transport layers with many bandgap states, the injected charge carriers would flow across the device via these trap states even if the device is still in an off-state under a bias lower than the threshold..$^{[4,75,94-111]}$ Nonuniformity in an LHP-NC film is another source of leakage current. The pinholes in a nonuniform LHP-NCs film act as a bypass that drains out charge carriers. Normally, both of these current density leaks are sustained at low levels and used to judge device quality. ${ }^{[4-7,59,95-97]}$ Pinholes can act as a parallel branch with a lower resistance, resulting in a localized higher density driving current. Therefore, a uniform LHP-NC film without pinholes is essential to ensure that all of the charge 
carriers flow across the emitters. Even in a high-quality device without any defects or pinholes, the charge carriers still have the opportunity to pass through the device without recombination as an overflow current, which is a type of leakage current at a high driving density. Thus, a charge carrier blocking layer is required to suppress this overflow current. ${ }^{[5,7,96,97,99]}$

The charge carrier balance in the recombination zone is another important factor in determining the EQE, which is consistent with the role played by the emissive film PLQY. ${ }^{4-7,59,94-111]}$ For an LHP-NC LED driven by imbalanced charge carriers in the recombination zone, the excess carrier species are themselves the loss, and the resulting interaction with excitons leads to an additional loss, even at a low driving current density, via the Auger nonradiative process. The comparable mobilities of electrons and the holes of LHP-NCs are a good feature for realizing balanced opposite charge carriers in the recombination zone. ${ }^{[25,26,92]}$ However, the field-dependent mobility of the matched functional layers makes it difficult to balance the opposite charge at all times under a changing bias. ${ }^{[145,146]}$

The advantage offered by a high defect tolerance is that trapinduced nonradiative recombination can be sustained at a negligible level, which has been confirmed by the near-unity PLQY of high-quality LHP-NCs. ${ }^{[4-7]}$ However, it is difficult to eliminate the Auger nonradiative recombination of multiexcitons at a high driving current density, which plays a significant role in EQE roll-off. ${ }^{[4-7,59,94-111,144]}$ Thus, adjusting the charge carrier transport capabilities of the matched function layers can result in balanced charge carrier injections into the LHP-NC layer, and a higher maximum EQE can be reached by suppressing the Auger process at a low driving current density level in most devices. ${ }^{[4-7,59,94-111,144]}$

\section{LHP-NC Color Converters for Display and Lighting}

The significant progress that has been made for LHP-NC-based LEDs in terms of luminous efficiency has been offset by their unsatisfactory operational stability, leaving the potential of this cutting-edge display technology uncertain. In principle, without directional ion migration driven by an applied electric field, LHP-NCs that work as color converter under only optical excitation are significantly more stable,$^{[9-11,147-162]}$ and all of the considerations concerning electrical features, e.g., charge carrier transport and injection, and related technical questions, e.g., film morphology and compatibility, can be neglected (Table 2). Moreover, quasi-2D LHP NCs, and especially the lead-free halide double perovskite NCs that are difficult to use in LEDs, can be used appropriately in various applications under optical excitation. Similar to consumer TVs using CdSe quantum dots color converters, LHPNCs can also be adopted to serve as superb color converters in full color displays in virtue of their high color purity, wide color gamut coverage, near-unity PLQY, and low cost (Figure 3c). ${ }^{[10,147,152]}$ Utilizing the color filters integrated with red, green, and blue $\mathrm{CsPbX}_{3}$ QDs, a liquid crystal display can exhibit an unprecedented color gamut over the NTSC standard under illumination by blue LEDs. ${ }^{[10]}$

Most commercialized white LEDs are based on yellow phosphor (YAG: $\mathrm{Ce}^{3+}$ ) integrated onto blue LED chips. However, the narrow linewidth of the blue component and the absence of a red component results in a typical color rendering index (CRI) of $\approx 80$ or below. Owing to the flexible tunability of their emissive wavelengths in the visible range, LHP-NCs can serve as color converters for white lighting applications with a high CRI (Table 2). ${ }^{[149,153,154]}$ The near-unity PLQY of LHP-NCs enables the resulting white LEDs to reach a high power efficiency over $70 \mathrm{~lm} \mathrm{~W}^{-1}$. $^{[154]}$ Classical YAG:Ce ${ }^{3+}$ white LEDs with a complementary red component from LHP-NCs phosphor exhibit efficient emission with a high CRI above 90 (Figure 3d). ${ }^{[148,149,154,161]}$ Another scenario for making white LEDs is using multicolor LHP-NC converters to completely replace the YAG: $\mathrm{Ce}^{3+}$ phosphor; however, the narrow band emission of LHP-NCs will result in a lower CRI of the resulting white LEDs compared to the YAG:Ce/LHP-NCs hybrid phosphor LEDs, unless additional color components are used. ${ }^{[157,158]}$

In comparison to the role of emitters in LEDs, lead-free double perovskite $\mathrm{NCs}$, e.g., $\mathrm{Cs}_{2} \mathrm{AgInCl}_{6}$, exhibit superior performance as phosphor in white LED because of their broad band emission. ${ }^{[9,159,160,162]}$ With a high PLQY of $86 \%$ and a superior operation lifetime of over $10000 \mathrm{~h}$ with continuous illumination by ultraviolet light in the ambient environment, ${ }^{[9]}$ such lead-free double perovskite NCs could be used in general lighting. A more significant concern with lead-free perovskite converters is environmental effects. ${ }^{[9]}$ The exclusion of toxic lead- and heavy lanthanide-based phosphors in white LEDs would be a bright prospect in lighting applications.

The prompt exciton radiative recombination and rapid photon response of LHP-NC phosphors lead to their potential application in short-distance wireless data transmission technology, i.e., Li-Fi, an emerging branch of lighting applications for white LEDs (Figure 3e). ${ }^{[12,13]}$ Mixing $\mathrm{CsPbBr}_{3}$ NCs with conventional red phosphors produces white color converters that exhibit a modulation bandwidth of $491 \mathrm{MHz}$ and a high data transmission rate of up to 2 Gbit $\mathrm{s}^{-1}$. ${ }^{[13]}$ Meanwhile, as qualified lighting devices, the corresponding white LEDs demonstrate an efficient warm white emission with a CRI of $\approx 89 .{ }^{[12]}$

To protect N-LHP phosphors from ambient moisture and oxygen, polymer or inorganic matrices are used to disperse the LHP-NCs, which can also prevent a emissive spectra shift from an ion migration of LHP-NC phosphors with alloyed halogen species. ${ }^{[149,161,162]}$

Table 2. WLEDs using LHP-NCs as color phosphor.

\begin{tabular}{|c|c|c|c|c|}
\hline Color converter & Efficiency $\left[\mathrm{Im} \mathrm{W} \mathrm{W}^{-1}\right]$ & CRI & $\mathrm{CCT}[\mathrm{K}]$ & Refs. \\
\hline Blue LED+CsPbBr ${ }_{3-x} \mathrm{I}_{x}$ QDs+YAG:Ce ${ }^{3+}$ & 58 & 90 & 5907 & [149] \\
\hline Blue LED+CsPbBr ${ }_{3} \mathrm{NCs}+\mathrm{BaCe}_{4} \mathrm{O}_{9}: \mathrm{Eu}^{3+}$ & 38.8 & - & 4033 & [11] \\
\hline Blue LED+CsPbBr ${ }_{1-x} \mathrm{I}_{x}$ QDs/silica+PiC & 75.2 & 92 & 5198 & [161] \\
\hline Blue LED+CsPbBr $\left.{ }_{1.2}\right|_{1.8}$ QDs+YAG:Ce ${ }^{3+}$ & 78.4 & 92 & 4222 & [154] \\
\hline Blue LED+CsPb $\left(\mathrm{Br}_{0.4} \mathrm{I}_{0.6}\right)_{3}+$ YAG: $\mathrm{Ce}^{3+}$ & 46.45 & 90.3 & 3897 & [148] \\
\hline UV LED+FA $\mathrm{AMA}_{1-x} \mathrm{PbBr}_{3}$ QDs & 40.2 & 85 & - & [157] \\
\hline
\end{tabular}


Table 3. Lasing characteristics from LHP-NCs.

\begin{tabular}{|c|c|c|c|c|c|}
\hline Materials & Pumping source & Threshold & Wavelength & Temperature $[\mathrm{K}]$ & Refs. \\
\hline $\mathrm{CsPbX}_{3} \mathrm{NCs}$ & $400 \mathrm{~nm}, 100 \mathrm{fs}$ & $5 \pm 1 \mu \mathrm{Jcm}-2$ & $440-700 \mathrm{~nm}$ & 293 & [15] \\
\hline $\mathrm{CsPbCl}_{3}$ NPLs & $375 \mathrm{~nm}, 100 \mathrm{fs}$ & $12 \mu \mathrm{J} \mathrm{\textrm {cm } ^ { - 2 }}$ & $\approx 2.90 \mathrm{eV}$ & 293 & [17] \\
\hline $\mathrm{CsPbX}_{3} \mathrm{QDs}$ & $400 \mathrm{~nm}, 100 \mathrm{fs}$ & $0.59-7.89 \mathrm{~m} / \mathrm{cm}^{-2}$ & $400-700 \mathrm{~nm}$ & 293 & [16] \\
\hline $\mathrm{MAPbBr}_{3} \mathrm{NCs}$ & $405 \mathrm{~nm}, \mathrm{CW}$ & $15 \mathrm{~W} \mathrm{~cm}^{-2}$ & $538.7 \mathrm{~nm}$ & 293 & [170] \\
\hline $\mathrm{CsPbX}_{3} \mathrm{NCs}$ & $400 \mathrm{~nm}, 100 \mathrm{fs}$ & $9 \mu \mathrm{cm}^{-2}$ & $470-620 \mathrm{~nm}$ & 293 & [14] \\
\hline $\mathrm{CsPbBr}_{3} \mathrm{NCs}$ & $400 \mathrm{~nm}, 100 \mathrm{fs}$ & $471 \mu \mathrm{J} \mathrm{\textrm {cm } ^ { - 2 }}$ & Random lasing & 293 & [164] \\
\hline
\end{tabular}

\section{Lasing Based on LHP-NCs}

In addition to efficient luminescence, LHP-NCs also exhibit good coherent emissions, which can be exploited in conventional lasing based on population inversion and low-threshold exciton-polariton lasing; this exploitation is due to their high absorption, high PLQY, and low nonradiative recombination loss endowed by excellent defect tolerance (Table 3). ${ }^{[14-17,163-170]}$ The emission wavelength of the laser can be tuned over the entire visible range by changing components and structures of LHP-NCs. Using a natural whispering-gallery-mode optical resonant cavity with a polygonal morphology or a natural FabryPérot optical cavity with single-crystal nanowires, mesoscopic LHPs exhibit low-threshold lasing. ${ }^{[14,163]}$ By comparison, an additional optical resonant cavity or feedback structure needs to be incorporated into LHP-NCs with dimensions of only several nanometers to stimulate coherent emission, ${ }^{[14,15,164,166]}$ which does not offer a distinct advantage, e.g., a threshold, over bulky LHPs in population inversion lasing. ${ }^{[14-16,163,164,166-170]}$

\subsection{Gain Medium of LHP-NCs for Population Inversion Lasing}

In comparison to bulky LHPs, LHP-NCs demonstrate a peak PLQY at lower excitation densities, followed by a drop in the PLQY at higher excitation densities, which is consistent with the operating conditions of the resulting LEDs. ${ }^{[59,69]}$ However, the threshold for population inversion lasing of LHP-NCs is higher by orders of magnitude than for luminescence. ${ }^{[69]}$ Thus, LHP-NCs do not appear to provide a good gain medium for low threshold population inversion lasing compared to bulky counterparts. ${ }^{[69]}$ From practical perspective, LHP-NCs with smaller grain sizes of approximately several nanometers and facile solution processing exhibit good compatibility with designed optical resonant cavities or other photonic structures for application. ${ }^{[14,15,164,166]}$

Because of the excellent defect tolerance, the theoretical threshold loss caused from trap-assisted nonradiative recombination is low, however, in population inversion lasing, the gain medium still need to be exposed to a high pumping flu-

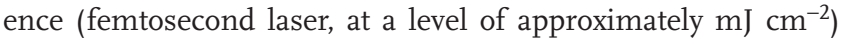
to reach the threshold of population inversion, ${ }^{14-16,163,164,166-170] ~}$ which is a significant challenge to the stability of LHP-NCs at room temperature. The superb stability of $\mathrm{CsPbr}_{3} \mathrm{NCs}$ at high temperature up to $690 \mathrm{~K}$ makes them more suitable for such lasing applications. ${ }^{[14-17,164,171]}$ Thus far, because competing Auger nonradiative recombination occurs on the order of picoseconds, lasers with ultrashort pulses in the femtosecond range have been used to pump the gain medium of LHPNCs. ${ }^{[14-16,163,164,166-170]}$ Yet a breakthrough for the realization of optically pumped continuous wave lasing operating at room temperature is required. It is difficult to observe continuous wave lasing from LHP-NCs than pulse lasing because of the investable Auger nonradiative recombination. [14-16,163,164,166-170] Similarly, in potential electrically pumped LHP-NC lasers, continuous electrical excitation also results in significant Auger nonradiative recombination that can be aggravated by injected charge carriers, especially the excess charge carriers caused by imbalanced injection. In addition, leakage current and overflow current lead to a much higher threshold than for optically pumped counterparts. The Joule heating generated by high driving current density (up to $\mathrm{kA} \mathrm{cm}^{-2}$ ) and other heating created from various energy losses result in a sustaining challenge to the thermal stability of LHP-NCs and EQE roll-off. ${ }^{[143]}$ Similar to the LEDs mentioned above, LHP-NCs can be degraded by an applied electric field. Most of the functional layers used in LHP-NC LEDs are organic molecules; thus, the charge carrier transport capability and thermal tolerance of these devices are too low to support a high driving current density laser.

\subsection{Exciton-Polariton Lasing Based on LHP-NCs}

In theory, exciton-polariton lasing provides an alternative pathway to low threshold coherent emission without population inversion. ${ }^{[17,172-178]}$ In electrically pumped InGaAs excitonpolariton lasers, the threshold of exciton-polariton lasing is 1-3 orders of magnitude lower than that for population inversion lasing in the same diode. ${ }^{[175,176]}$ The sufficiently strong coupling between an exciton and a photon leads to a composite boson known as an exciton-polariton, a new quantum superposition state containing two branches that consist of both light and matter. ${ }^{[172-178]}$ Both the lower and upper branches are mixtures of the original photon and exciton states. ${ }^{[172-178]}$ Above a critical particle density, a macroscopic number of bosons undergoes a nonequilibrium cooling process, i.e., stimulated scattering caused by repulsive interactions, and then accumulates in the ground state, i.e., a single quantum state, of the lower excitonpolariton branch, which is regarded as Bose-Einstein condensation. ${ }^{[172-178]}$ Eventually, a large number of condensed exciton-polaritons decay from the single state by leaking coherent photons. ${ }^{[172-178]}$

The extremely light effective mass of $\approx 10^{-4} m_{\mathrm{e}}\left(m_{\mathrm{e}}\right.$ is the electron mass) endowed by the partial light nature of the particle 


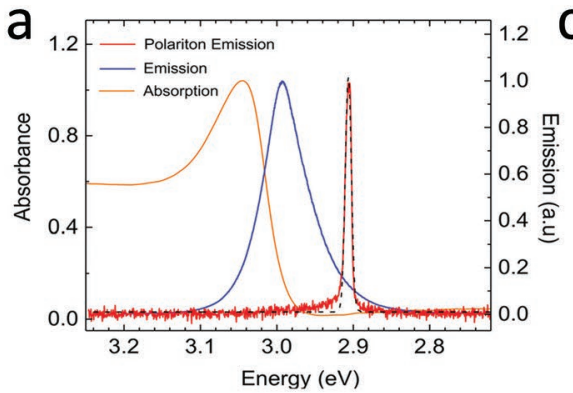

b

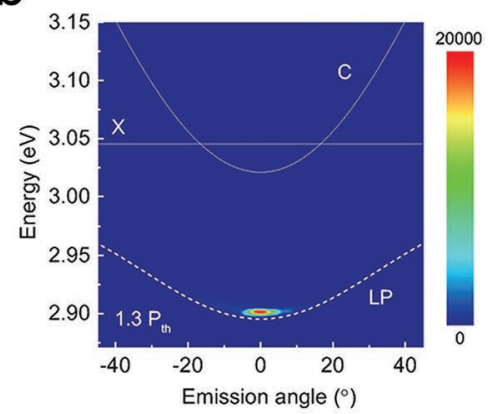

C

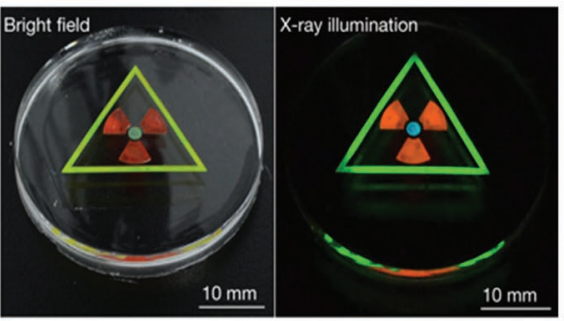

d

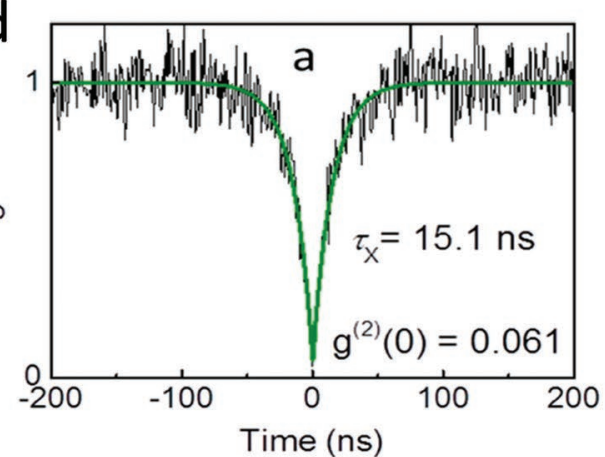

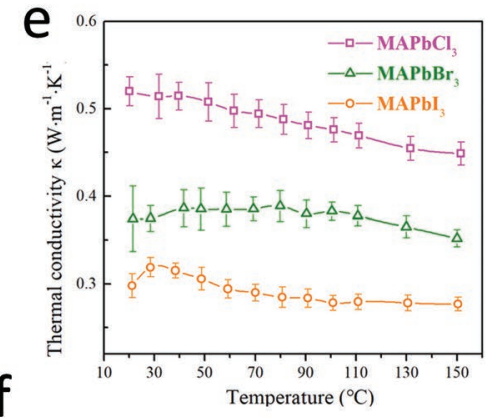

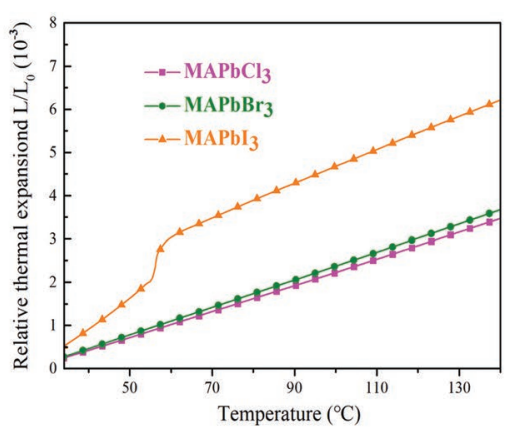

Figure 4. a) PL, absorption, and exciton-polariton emission spectra of the $\mathrm{CsPbCl}_{3} \mathrm{NPLs}$. Reproduced with permission. ${ }^{[17]}$ Copyright 2017, American Chemical Society. b) Power-dependent angle-resolved PL spectra. Angle-resolved PL spectrum measured at 1.3 Pth (Pth is threshold). The ground state near $k_{\|}=0$ is greatly occupied, resulting in a sharp increase in intensity. Reproduced with permission. ${ }^{[17]}$ Copyright 2017 , American Chemical Society. c) Multicolor X-ray scintillation pattern from LHP NCs with different halide species. Reproduced with permission. ${ }^{[19]}$ Copyright 2018, Springer Nature. d) The second-order temporal correlation function of PL intensity of a single CsPbl ${ }^{3}$ QD under illumination of continuous wave lasing. Reproduced

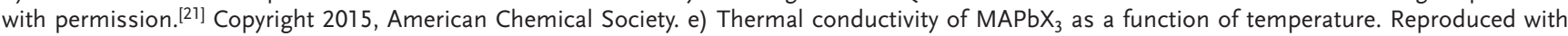
permission. ${ }^{[189]}$ Copyright 2018, American Chemical Society. $f$ ) Temperature dependence of thermal expansion $d L / L_{0}$ of MAPbX ${ }_{3}$. Reproduced with permission. ${ }^{[189]}$ Copyright 2018, American Chemical Society.

results in a long thermal De Broglie wavelength that makes the Bose-Einstein condensation of LHP-NCs exciton-polaritons possible even at room temperature. ${ }^{[17,175,176]}$ A vertical FabryPérot resonant cavity with the planar structure of a LHP-NC layer sandwiched by two distributed Bragg reflectors has been used in exciton-polariton lasing. ${ }^{[17]}$ Exciton-photon coupling can occur if the placement of an LHP-NC film is well-matched with an antinode of the confined photon mode of the optical resonant cavity at almost equal exciton and cavity photon energies. ${ }^{[17,179,180]}$ Eventually, an exciton-polariton lasing with a threshold of $12 \mu \mathrm{J} \mathrm{cm}^{-2}$ was observed (Figure $4 \mathrm{a}, \mathrm{b}$ ). The verticalcavity surface-emitting structure similar to LHP-NC LEDs shows good compatibility with a potential electrically driven laser requiring external charge carrier injection.

\section{More Applications of LHP-NC Emitters}

\subsection{LHP-NCs for lonizing Radiation Detection}

In addition to LEDs, color converters, and coherent emission, other nonclassic applications using LHP-NC emitters have been explored, e.g., direct detection for X-ray imaging and $\gamma$-ray energy spectroscopy. ${ }^{[18,19,181,182]}$ After high energy X-ray photons with energies of up to dozens of $\mathrm{keV}$ are absorbed, high-energy electrons and holes are ionized and then relax to the conduction and valence band edges of LHP-NCs, respectively. ${ }^{[18,19,181,182]}$
The LHP-NC scintillator subsequently emits visible light that can be recorded by an arrayed photodetector (Figure 4c). For $\mathrm{X}$-ray photons with energies of approximately dozens of $\mathrm{keV}$, the photoelectric effect is a predominant mechanism for converting photons into charge carriers, which depends on the effective atomic number $\left(Z_{\text {eff }}\right)$ of the absorber; therefore, LHP-NCs, e.g., $\mathrm{CsPBr}_{3}$ with a $Z_{\text {eff }}$ of 65.9 , show a high absorption of photons at this level of $\mathrm{keV}^{\left[{ }^{[19]}\right.}$ With a high atomic number of $82, \mathrm{~Pb}$ plays a critical role in LHP-NC X-ray scintillators. ${ }^{[19]}$ The nanoscale dimensions of LHP-NCs produce weak light scattering, such that the resulting thin film detector has a high spatial resolution. With a large X-ray stopping power and a high emissive quantum yield, $13 \mathrm{nGy} \mathrm{s}^{-1}$ the sensitivity of LHP-NCs thin film scintillators is comparable to the sensitivity of commercial bulk scintillators. ${ }^{[19]}$ LHPNCs exhibit good stability, which may be attributed to self-healing via significant ion migration. ${ }^{[19,182]}$ Similar to X-ray imaging, a strong stopping power caused by the large atomic number of lead also plays a significant role in $\gamma$-ray detection. ${ }^{[182-184]}$ These features extend the potential applications of LHPs-NCs to ultrashortwave optical imaging in space, wherein high energy photons could be converted into visible photons to form high-resolution

\subsection{LHP-NCs Single Photon Emitters}

Single photon emitters play an important role in flourishing quantum technologies, and some studies have been conducted 
on the potential of using individual LHP-NCs as single photon emitters. ${ }^{[20,21,58]}$ An ideal single photon emitter releases exactly one photon at a time, and the photons are coherent. ${ }^{[20,21,58,185-187]}$ The $\mathrm{CsPbX}_{3}$ quantum dots emit prompt radiation at the level of hundreds of picoseconds, which meets the requirement of $T_{2}=2 T_{1}$ for a single photon emitter, ${ }^{[20]}$ where $T_{1}$ is the spontaneous emission lifetime of the emitter, and $T_{2}$ is the optical coherence time. The second-order temporal correlation function $g^{(2)}(0)$, of approximately $6 \%$ indicates a high purity of single photon emissions from the individual $\mathrm{CsPbX}_{3}$ quantum dots dispersed in a poly(methyl methacrylate) (PMMA) matrix (Figure 4d). ${ }^{[21]}$ Fast Auger nonradiative recombination can effectively quench the multiphoton emissions from biexcitons and multiexcitons, which is a significant contribution to strong photon antibunching. ${ }^{[21,58]}$ In addition, the features of a narrow PL linewidth, highly stable spectra, and low emission blinking all contribute to the high quality of the single photon emissions. ${ }^{[20,21]}$

\section{Stability of LHP-NC Applications}

Impressive progress has been made in using LHP-NCs in various light generation applications; however, the stability of LHP-NCs will play a critical and even ultimate role in determining their commercial prospects. In this section, commonly held views about LHP-NC applications will be discussed. The factors influencing their stability of LHP-NCs and the resulting applications mainly result from the material characteristics of LHP-NCs. The stability issue for optical excitation applications is mainly from the degradation of LHP-NCs that results from their ionic bonding, high density of vacancy defects, and resulting ion migration. ${ }^{[52,121,124,127,128]}$ Which has also been observed in metallic oxide perovskites. ${ }^{[188]}$ Furthermore, this ionic conductivity of LHP-NCs can be boosted by light illumination. ${ }^{[52,129]}$ Ion migration can be effectively suppressed in high-quality LHPs with a low density of vacancy defects; ${ }^{[121,125]}$ however, it is difficult to apply this method to LHP-NCs, which have a large surface area but a small volume. ${ }^{[121]}$ Layered quasi2D LHP-NCs that are dispersed in a matrix at a low concentration can enhance the stability of optically driven applications against ion migration and the ambient atmosphere, especially under moist conditions.

In comparison, stability considerations for electrically driven devices are more complex because of the participation of injected charge carriers and the applied electrical field. A proposed predominant reason for this instability is still ion migration under an applied electrical field. Layered LHP-NCs provide an alternative means of suppressing ion migration by sandwiching $\left[\mathrm{PbX}_{6}\right]^{4-}$ cells with insulating ligands, that also block electron transport. A similar concept has been used in application of various core-cell structures or coating technology to suppress ion migration in LHP-NCs, thereby enhancing stability. A semiconductive matrix can be used to disperse LHP-NCs, wherein the inhomogeneous structure can suppress ion migration, while reserving a pathway for electron transport.

Superior electrical conductivity combined with ultralow thermal conductivity could make LHP-NCs a potential candidate for thermoelectric applications (Figure 4e,f); ${ }^{[189,190]}$ how- ever, the mechanical stress from the structural instability of polymorphism limits the operational stability of LHP-NC applications, especially for electrically driven devices of LEDs and prospective lasers because of the high amount of heating generated by a high driving current density. ${ }^{[189,190]}$ For a complete device, high-quality device encapsulation is essential to protect LHP-NCs and other functional layers against oxygen and moisture in the ambient atmosphere.

\section{Conclusion and Prospects}

In the past few years, superior features for light generation and facile solution processing have resulted in impressive progress in using LHP-NCs for numerous applications, that demonstrate equal or even higher performance than their counterpart materials. Among all of these applications, LHP-NC color converters, especially lead-free LHP-NCs that are economical and environmentally friendly, have the best prospect for commercialization in full color displays and lighting. Attempts at producing a slow device decay for highly efficient LEDs using LHP-NC emitters remain unsuccessful. In lasing applications, except an alternative pathway for stimulated coherent emission, LHP-NCs provide another form of coherent spontaneous emissions at room temperature via exciton-polaritons that possess a low threshold without population inversion and is promising for creating an electrically driven laser. Other novel applications of LHP-NCs have been discovered, e.g., high energy photon imaging and quantum light emitters, and more applications will be discovered in the future.

However, there are still some obstacles to the mass production of LHP-NC light generation applications. The intrinsic migration of A-cations and halide anions, especially when driven by an applied electrical field, results in the degradation of LHPNCs and low operational stability of the resulting applications. In addition, the environmental hazard posed by toxic lead also hinders the prospects for real-life LHP-NC applications. Various studies have been conducted to address these two problems. Lead-free double perovskites have been developed that possess superior features for use as color converters; however, deposition techniques make these materials difficult to use in electrically driven devices. An additional consideration for the commercial application of LHP-NC LEDs in information displays is the deposition of pixels with micrometre dimensions, which is a common obstacle for other solution-processed LED display technologies, e.g., CdSe quantum dots, LEDs, and polymer LEDs.

\section{Acknowledgements}

This research was supported by the National Research Foundation, Prime Minister's Office, Singapore under its competitive Research Programme (CRP Award No. NRF-CRP14-2014-03). H.V.D. gratefully acknowledges the financial support from an NRF Investigatorship grant NRF-NRFI2016-08 and additional support from TUBA.

\section{Conflict of Interest}

The authors declare no conflict of interest. 


\section{Keywords}

perovskite light generation nanocrystals

Received: April 25, 2019

Revised: August 22, 2019

Published online: October 25, 2019

[1] X. Hong, T. Ishihara, A. V. Nurmikko, Solid State Commun. 1992 $84,657$.

[2] T. Hattori, T. Taira, M. Era, T. Tsutsui, S. Saito, Chem. Phys. Lett. 1996, 254, 103

[3] K. Chondroudis, D. B. Mitzi, Chem. Mater. 1999, 11, 3028.

[4] K. Lin, J. Xing, L. N. Quan, F. P. G. Arquer, X. Gong, J. Lu, L. Xie, W. Zhao, D. Zhang, C. Yan, W. Li, X. Liu, Y. Lu, J. Kirman, E. H. Sargent, Q. Xiong, Z. Wei, Nature 2018, 562, 245.

[5] J. Song, T. Fang, J. Li, L. Xu, F. Zhang, B. Han, Q. Shan, H. Zeng, Adv. Mater. 2018, 30, 1805409.

[6] B. Zhao, S. Bai, V. Kim, R. Lamboll, R. Shivanna, F. Auras, J. M. Richter, L. Yang, L. Dai, M. Alsari, X. She, L. Liang, J. Zhang, S. Lilliu, P. Gao, H. J. Snaith, J. Wang, N. C. Greenham, R. H. Friend, D. Di, Nat. Photonics 2018, 12, 783.

[7] T. Chiba, Y. Hayashi, H. Ebe, K. Hoshi, J. Sato, S. Sato, Y. Pu, S. Ohisa, J. Kido, Nat. Photonics 2018, 12, 681.

[8] Z. Li, Z. Chen, Y. Yang, Q. Xue, H. Yip, Y. Cao, Nat. Commun. 2019, 10, 1027

[9] J. Luo, X. Wang, S. Li, J. Liu, Y. Guo, G. Niu, L. Yao, Y. Fu, L. Gao, Q. Dong, C. Zhao, M. Leng, F. Ma, W. Liang, L. Wang, S. Jin, J. Han, L. Zhang, J. Etheridge, J. Wang, Y. Yan, E. H. Sargent, J. Tang, Nature 2018, 563, 541.

[10] Y. H. Ko, M. Jalalah, S. J. Lee, J. G. Park, Sci. Rep. 2018, 8, 12881.

[11] H. Ding, W. Liu, Y. Zheng, C. Li, H. Jiang, X. Wang, J. Mater. Chem. C 2019, 7, 1690.

[12] I. Dursun, C. Shen, M. R. Parida, J. Pan, S. P. Sarmah, D. Priante, N. Alyami, J. Liu, M. I. Saidaminov, M. S. Alias, A. L. Abdelhady, T. K. Ng, O. F. Mohammed, B. S. Ooi, O. M. Bakr, ACS Photonics 2016, 3, 1150 .

[13] S. Mei, X. Liu, W. Zhang, R. Liu, L. Zheng, R. Guo, P. Tian, ACS Appl. Mater. Interfaces 2018, 10, 5641.

[14] Y. Wang, X. Li, J. Song, L. Xiao, H. Zeng, H. Sun, Adv. Mater. 2015, 27, 7101.

[15] S. Yakunin, L. Protesescu, F. Krieg, M. I. Bodnarchuk, G. Nedelcu, M. Humer, G. D. Luca, M. Fiebig, W. Heiss, M. V. Kovalenko, Nat. Commun. 2015, 6, 8056.

[16] X. Tang, Z. Hu, W. Chen, X. Xing, Z. Zang, W. Hu, J. Qiu, J. Du, Y. Leng, X. Jiang, L. Mai, Nano Energy 2016, 28, 462.

[17] R. Su, C. Diederichs, J. Wang, T. C. H. Liew, J. Zhao, S. Liu, W. Xu, Z. Chen, Q. Xiong, Nano Lett. 2017, 17, 3982.

[18] J. H. Heo, D. H. Shin, J. K. Park, D. H. Kim, S. J. Lee, S. H. Im, Adv. Mater. 2018, 30, 1801743 .

[19] Q. Chen, J. Wu, X. Ou, B. Huang, J. Almutlaq, A. A. Zhumekenov, X. Guan, S. Han, L. Liang, Z. Yi, J. Li, X. Xie, Y. Wang, Y. Li, D. Fan, D. B. L. Teh, A. H. All, O. F. Mohammed, O. M. Bakr, T. Wu, M. Bettinelli, H. Yang, W. Huang, X. Liu, Nature 2018, 561, 88.

[20] H. Utzat, W. Sun, A. E. K. Kaplan, F. Krieg, M. Ginterseder, B. Spokoyny, N. D. Klein, K. E. Shulenberger, C. F. Perkinson, M. V. Kovalenko, M. G. Bawendi, Science 2019, 363, 1068.

[21] Y. S. Park, S. Guo, N. S. Makarov, V. I. Klimov, ACS Nano 2015, 9, 10386.

[22] J. Xing, F. Yan, Y. Zhao, S. Chen, H. Yu, Q. Zhang, R. Zeng, H. V. Demir, X. W. Sun, Q. H. Xiong, ACS Nano 2016, 10, 6623.

[23] X. Du, G. Wu, J. Cheng, H. Dang, K. Ma, Y. Zhang, P. Tan, S. Chen, RSC Adv. 2017, 7, 10391.
[24] A. Sadhanala, S. Ahmad, B. Zhao, N. Giesbrecht, P. M. Pearce, F. Deschler, R. Hoye, K. Gödel, T. Bein, P. Docampo, S. Dutton, M. Volder, R. Friend, Nano Lett. 2015, 15, 6095.

[25] L. M. Herz, ACS Energy Lett. 2017, 2, 1539.

[26] C. Motta, F. El-Mellouhi, S. Sanvito, Sci. Rep. 2015, 5, 12746.

[27] J. Kang, L. W. Wang, J. Phys. Chem. Lett. 2017, 8, 489.

[28] J. S. Manser, J. A. Christians, P. V. Kamat, Chem. Rev. 2016, 116 , 12956.

[29] F. Liu, Y. Zhang, C. Ding, S. Kobayashi, T. Izuishi, N. Nakazawa, T. Toyoda, T. Ohta, S. Hayase, T. Minemoto, K. Yoshino, S. Dai, Q. Shen, ACS Nano 2017, 11, 10373.

[30] H. Huang, L. Polavarapu, J. A. Sichert, A. S. Susha, A. S. Urban, A. L. Rogach, NPG Asia Mater. 2016, 8, e328.

[31] L. Protesescu, S. Yakunin, M. I. Bodnarchuk, F. Krieg, R. Caputo, C. H. Hendon, R. X. Yang, A. Walsh, M. V. Kovalenko, Nano Lett. 2015, 15, 3692

[32] V. Srivastava, W. Liu, E. M. Janke, V. Kamysbayev, A. S. Filatov, C. J. Sun, B. Lee, T. Rajh, R. D. Schaller, D. V. Talapin, Nano Lett. 2017, 17, 2094.

[33] M. V. Kovalenko, L. Protesescu, M. I. Bodnarchuk, Science 2017, $358,745$.

[34] M. C. Weidman, A. J. Goodman, W. A. Tisdale, Chem. Mater. 2017, 29, 5019.

[35] J. A. Sichert, Y. Tong, N. Mutz, M. Vollmer, S. Fischer, K. Z. Milowska, R. G. Cortadella, B. Nickel, C. Cardenas-Daw, J. K. Stolarczyk, A. S. Urban, J. Feldmann, Nano Lett. 2015, 15, 6521.

[36] Y. Bekenstein, B. A. Koscher, S. W. Eaton, P. Yang, A. P. Alivisatos, J. Am. Chem. Soc. 2015, 137, 16008.

[37] Z. Y. Zhu, Q. Q. Yang, L. F. Gao, L. Zhang, A. Y. Shi, C. L. Sun, Q. Wang, H. L. Zhang, J. Phys. Chem. Lett. 2017, 8, 1610.

[38] B. Ai, C. Liu, J. Wang, J. Xie, J. Han, X. Zhao, J. Am. Ceram. Soc. 2016, 99, 2875

[39] X. Di, Z. Hu, J. Jiang, M. He, L. Zhou, W. Xiang, X. Liang, Chem. Commun. 2017, 53, 11068

[40] L. Meng, C. Yang, J. Meng, Y. Wang, Y. Ge, Z. Shao, G. Zhang, A. L. Rogach, H. Zhong, Nano Res. 2019, 12, 1411.

[41] Q. Zhang, H. Nan, Y. Zhou, Y. Gu, M. Tai, Y. Wei, F. Hao, J. Li, D. Oron, H. Lin, J. Mater. Chem. C 2019, 7, 6795.

[42] J. Xing, Y. Zhao, M. Askerka, L. N. Quan, X. Gong, W. Zhao, J. Zhao, H. Tan, G. Long, L. Gao, Z. Yang, O. Voznyy, J. Tang, Z. H. Lu, Q. Xiong, E. H. Sargent, Nat. Commun. 2018, 9, 3541.

[43] M. Do, I. Kim, M. A. Kolaczkowski, J. Kang, G. A. Kamat, Z. Yuan, N. S. Barchi, L. Wang, Y. Liu, M. J. Jurow, C. M. Sutter-Fella, Nanoscale 2019, 11, 17262.

[44] S. Wang, C. Bi, J. Yuan, L. Zhang, J. Tian, ACS Energy Lett. 2018, $3,245$.

[45] J. Sun, J. Yang, J. I. Lee, J. H. Cho, M. S. Kang, J. Phys. Chem. Lett. 2018, 9, 1573.

[46] G. Volonakis, A. A. Haghighirad, R. L. Milot, W. H. Sio, M. R. Filip, B. Wenger, M. B. Johnston, L. M. Herz, H. J. Snaith, F. Giustino, J. Phys. Chem. Lett. 2017, 8, 772.

[47] M. Leng, Y. Yang, K. Zeng, Z. Chen, Z. Tan, S. Li, J. Li, B. Xu, D. Li, M. P. Hautzinger, Y. Fu, T. Zhai, L. Xu, G. Niu, S. Jin, J. Tang, Adv. Funct. Mater. 2018, 28, 1704446.

[48] B. Philippe, T. J. Jacobsson, J. P. Correa-Baena, N. K. Jena, A. Banerjee, S. Chakraborty, U. B. Cappel, R. Ahuja, A. Hagfeldt, M. Odelius, H. Rensmo, J. Phys. Chem. C 2017, 121, 26655.

[49] S. Meloni, G. Palermo, N. Ashari-Astani, M. Grätzelb, U. Rothlisberger, J. Mater. Chem. A 2016, 4, 15997.

[50] X. Li, Y. Wu, S. Zhang, B. Cai, Y. Gu, J. Song, H. Zeng, Adv. Funct. Mater. 2016, 26, 2435.

[51] W. Tress, J. Phys. Chem. Lett. 2017, 8, 3106.

[52] G. Y. Kim, A. Senocrate, T. Y. Yang, G. Gregori, M. Grätzel, J. Maier, Nat. Mater. 2018, 17, 445 
[53] X. Liang, R. W. Baker, K. Wu, W. Deng, D. Ferdani, P. S. Kubiak, F. Marken, L. Torrente-Murciano, P. J. Cameron, React. Chem. Eng. 2018, 3, 640.

[54] S. Govinda, B. P. Kore, M. Bokdam, P. Mahale, A. Kumar, S. Pal, B. Bhattacharyya, J. Lahnsteiner, G. Kresse, C. Franchini, A. Pandey, D. D. Sarma, J. Phys. Chem. Lett. 2017, 8, 4113.

[55] Y. J. Choi, L. Debbichi, D. Lee, N. Park, H. Kim, D. Kim, J. Phys. Chem. Lett. 2019, 10, 2135.

[56] H. Huang, M. I. Bodnarchuk, S. V. Kershaw, M. V. Kovalenko, A. L. Rogach, ACS Energy Lett. 2017, 2, 2071.

[57] M. Pandey, K. W. Jacobsen, K. S. Thygesen, J. Phys. Chem. Lett. 2016, 7, 4346,

[58] Q. A. Akkerman, G. Rainò, M. V. Kovalenko, L. Manna, Nat. Mater. 2018, 17, 394.

[59] F. Yan, J. Xing, G. Xing, L. Quan, S. T. Tan, J. Zhao, R. Su, L. Zhang, S. Chen, Y. Zhao, A. Huan, E. H. Sargent, Q. Xiong, H. V. Demir, Nano Lett. 2018, 18, 3157.

[60] Y. H. Kima, H. Choa, T. W. Lee, Proc. Natl. Acad. Sci. USA 2016, 113,11694

[61] H. Cho, S. H. Jeong, M. H. Park, Y. H. Kim, C. Wolf, C. L. Lee, J. H. Heo, A. Sadhanala, N. Myoung, S. Yoo, S. H. Im, R. H. Friend, T. W. Lee, Science 2015, 350, 1222.

[62] Y. Yang, D. P. Ostrowski, R. M. France, K. Zhu, J. Lagemaat, J. M. Luther, M. C. Beard, Nat. Photonics 2016, 10, 53.

[63] M. Saba, M. Cadelano, D. Marongiu, F. Chen, V. Sarritzu, N. Sestu, C. Figus, M. Aresti, R. Piras, A. G. Lehmann, C. Cannas, A. Musinu, F. Quochi, A. Mura, G. Bongiovanni, Nat. Commun. 2014, 5, 5049.

[64] M. B. Johnston, L. M. Herz, Acc. Chem. Res. 2016, 49, 146.

[65] S. Rana, K. Awasthi, S. S. Bhosale, E. W.-G. Diau, N. Ohta, J. Phys. Chem. C 2019, 123, 19927.

[66] B. Wu, H. Yuan, Q. Xu, J. A. Steele, D. Giovanni, P. Puech, J. Fu, Y. F. Ng, N. F. Jamaludin, A. Solanki, S. Mhaisalkar, N. Mathews, M. B. J. Roeffaers, M. Grätzel, J. Hofkens, T. C. Sum, Nat. Commun. 2019, 10, 484.

[67] F. Ambrosio, J. Wiktor, F. D. Angelis, A. Pasquarello, Energy Environ. Sci. 2018, 11, 101.

[68] G. Xing, N. Mathews, S. S. Lim, N. Yantara, X. Liu, D. Sabba, M. Grätzel, S. Mhaisalkar, T. C. Sum, Nat. Mater. 2014, 13, 476.

[69] G. Xing, B. Wu, X. Wu, M. Li, B. Du, Q. Wei, J. Guo, E. K. L. Yeow, T. C. Sum, W. Huang, Nat. Commun. 2017, 8, 14558.

[70] V. Sarritzu, N. Sestu, D. Marongiu, X. Chang, Q. Wang, S. Masi, S. Colella, A. Rizzo, A. Gocalinska, E. Pelucchi, M. L. Mercuri, F. Quochi, M. Saba, A. Mura, G. Bongiovanni, Adv. Opt. Mater. 2018, 6, 1701254

[71] S. D. Stranks, V. M. Burlakov, T. Leijtens, J. M. Ball, A. Goriely, H. J. Snaith, Phys. Rev. Appl. 2014, 2, 034007.

[72] V. K. Ravi, A. Swarnkar, R. Chakraborty, A. Nag, Nanotechnology 2016, 27, 325708.

[73] H. C. Woo, J. W. Choi, J. Shin, S. H. Chin, M. H. Ann, C. L. Lee, J. Phys. Chem. Lett. 2018, 9, 4066.

[74] K. Wang, C. Wu, D. Yang, Y. Jiang, S. Priya, ACS Nano 2018, 12, 4919.

[75] B. J Bohn, Y. Tong, M. Gramlich, M. L. Lai, M. Döblinger, K. Wang, R. L. Z. Hoye, P. Müller-Buschbaum, S. D. Stranks, A. S. Urban, L. Polavarapu, J. Feldmann, Nano Lett. 2018, 18, 5231.

[76] S. Demchyshyn, J. M. Roemer, H. Groiß, H. Heilbrunner, C. Ulbricht, D. Apaydin, A. Böhm, U. Rütt, F. Bertram, G. Hesser, M. C. Scharber, N. S. Sariciftci, B. Nickel, S. Bauer, E. D. Głowacki, M. Kaltenbrunner, Sci. Adv. 2017, 3, el700738.

[77] Q. A. Akkerman, S. G. Motti, A. R. S. Kandada, E. Mosconi, V. D'Innocenzo, G. Bertoni, S. Marras, B. A. Kamino, L. Miranda, F. De Angelis, A. Petrozza, M. Prato, L. Manna, J. Am. Chem. Soc. 2016, 138, 1010.

[78] M. A. Becker, R. Vaxenburg, G. Nedelcu, P. C. Sercel, A. Shabaev, M. J. Mehl, J. G. Michopoulos, S. G. Lambrakos, N. Bernstein,
J. L. Lyons, T. Stöferle, R. F. Mahrt, M. V. Kovalenko, D. J. Norris, G. Rainò, A. L. Efros, Nature 2018, 553, 189.

[79] A. D. Wright, C. Verdi, R. L. Milot, G. E. Eperon, M. A. Pérez-Osorio, H. J. Snaith, F. Giustino, M. B. Johnston, L. M. Herz, Nat. Commun. 2016, 7, 11755.

[80] J. A. Steele, P. Puech, M. Keshavarz, R. Yang, S. Banerjee, E. Debroye, C. W. Kim, H. Yuan, N. H. Heo, J. Vanacken, A. Walsh, J. Hofkens, M. B. J. Roeffaers, ACS Nano 2018, 12, 8081.

[81] M. I. Saidaminov, A. L. Abdelhady, B. Murali, E. Alarousu, V. M. Burlakov, W. Peng, I. Dursun, L. Wang, Y. He, G. Maculan, A. Goriely, T. Wu, O. F. Mohammed, O. M. Bakr, Nat. Commun. 2015, 6, 7568.

[82] J. Butkus, P. Vashishtha, K. Chen, J. K. Gallaher, S. K. K. Prasad, D. Z. Metin, G. Laufersky, N. Gaston, J. E. Halpert, J. M. Hodgkiss, Chem. Mater. 2017, 29, 3644

[83] J. M. Richter, M. Abdi-Jalebi, A. Sadhanala, M. Tabachnyk, J. P. H. Rivett, L. M. Pazos-Outón, K. C. Gödel, M. Price, F. Deschler, R. H. Friend, Nat. Commun. 2016, 7, 13941.

[84] K. Miyata, D. Meggiolaro, M. T. Trinh, P. P. Joshi, E. Mosconi, S. C. Jones, F. D. Angelis, X. Y. Zhu, Sci. Adv. 2017, 3, e1701217.

[85] T. J. S. Evans, K. Miyata, P. P. Joshi, S. Maehrlein, F. Liu, X. Y. Zhu, J. Phys. Chem. C 2018, 122, 13724.

[86] X. Y. Zhu, V. Podzorov, J. Phys. Chem. Lett. 2015, 6, 4758

[87] C. Motta, S. Sanvito, J. Phys. Chem. C 2018, 122, 1361.

[88] A. Filippetti, A. Mattoni, Phys. Rev. B 2014, 89, 125203.

[89] G. Giorgi, J. Fujisawa, H. Segawa, K. Yamashita, J. Phys. Chem. Lett. 2013, 4, 4213.

[90] A. Miyata, A. Mitioglu, P. Plochocka, O. Portugall, J. T. W. Wang, S. D. Stranks, H. J. Snaith, R. J. Nicholas, Nat. Phys. 2015, 11, 582.

[91] L. M. Herz, J. Phys. Chem. Lett. 2018, 9, 6853.

[92] Y. Kang, S. Han, Phys. Rev. Appl. 2018, 10, 044013.

[93] J. Lim, M. T. Hörantner, N. Sakai, J. M. Ball, S. Mahesh, N. K. Noel, Y.-H. Lin, J. B. Patel, D. P. McMeekin, M. B. Johnston, B. Wenger, H. J. Snaith, Energy Environ. Sci. 2019, 12, 169.

[94] K. Sim, T. Jun, J. Bang, H. Kamioka, J. Kim, H. Hiramatsu, H. Hosono, Appl. Phys. Rev. 2019, 6, 031402.

[95] M. Lu, H. Wu, X. Zhang, H. Wang, Y. Hu, V. L. Colvin, Y. Zhang, W. W. Yu, ChemNanoMat 2019, 5, 313.

[96] X. Yang, X. Zhang, J. Deng, Z. Chu, Q. Jiang, J. Meng, P. Wang, L. Zhang, Z. Yin, J. You, Nat. Commun. 2018, 9, 570.

[97] Y. Yao, H. Yu, Y. Wu, Y. Lu, Z. Liu, X. Xu, B. Ma, Q. Zhang, S. Chen, W. Huang, ACS Omega 2019, 4, 9150.

[98] H. Lee, H. Kim, H. Cho, W. Cha, Y. Hong, Y. Kim, A. Sadhanala, V. Venugopalan, J. S. Kim, J. W. Choi, C. Lee, D. Kim, H. Yang, R. H. Friend, T. W. Lee, Adv. Funct. Mater. 2019, 29, 1901225

[99] Z. Xiao, R. A. Kerner, L. Zhao, N. L. Tran, K. M. Lee, T. W. Koh, G. D. Scholes, B. P. Rand, Nat. Photonics 2017, 11, 108.

[100] L. Zhang, X. Yang, Q. Jiang, P. Wang, Z. Yin, X. Zhang, H. Tan, Y. Yang, M. Wei, B. R. Sutherland, E. H. Sargent, J. You, Nat. Commun. 2017, 8, 15640.

[101] A. Perumal, S. Shendre, M. Li, Y. K. E. Tay, V. K. Sharma, S. Chen, Z. Wei, Q. Liu, Y. Gao, P. J. S. Buenconsejo, S. T. Tan, C. L. Gan, Q. Xiong, T. C. Sum, H. V. Demir, Sci. Rep. 2016, 6, 36733.

[102] S. T. Ochsenbein, F. Krieg, Y. Shynkarenko, G. Rainò, M. V. Kovalenko, ACS Appl. Mater. Interfaces 2019, 11, 21655.

[103] S. Kumar, T. Marcato, S. I. Vasylevskyi, J. Jagielski, K. M. Fromm, C. Shih, J. Mater. Chem. C 2019, 7, 8938.

[104] H. D. Lee, H. Kim, H. Cho, W. Cha, Y. Hong, Y. H. Kim, A. Sadhanala, V. Venugopalan, J. S. Kim, J. W. Choi, C. L. Lee, D. Kim, H. Yang, R. H. Friend, T. W. Lee, Adv. Funct. Mater. 2019, 29, 1901225.

[105] J. H. Park, A. Lee, J. C. Yu, Y. S. Nam, Y. Choi, J. Park, M. H. Song, ACS Appl. Mater. Interfaces 2019, 11, 8428.

[106] W. Deng, X. Jin, Y. Lv, X. Zhang, X. Zhang, J. Jie, Adv. Funct. Mater. 2019, 29, 1903861. 
[107] Y. Hassan, O. J. Ashton, J. H. Park, G. Li, N. Sakai, B. Wenger, A. Haghighirad, N. K. Noel, M. H. Song, B. R. Lee, R. H. Friend, H. J. Snaith, J. Am. Chem. Soc. 2019, 141, 1269.

[108] L. Zhang, Q. Zhang, X. Xing, Y. Jiang, T. He, Y. Huang, Z. Ma, J. Yang, M. Yuan, Chem NanoMat 2019, 5, 318.

[109] L. Wang, B. Liu, X. Zhao, H. V. Demir, H. Gu, H. Sun, ACS Appl. Mater. Interfaces 2018, 10, 19828.

[110] B. K. Chandran, S. A. Veldhuis, X. Y. Chin, A. Bruno, N. Yantara, X. Chen, S. Mhaisalkar, Phys. Chem. Chem. Phys. 2018, 20, 5918.

[111] S. Kumar, J. Jagielski, T. Tian, N. Kallikounis, W. Lee, C. Shih, ACS Energy Lett. 2019, 4, 118.

[112] M. Petrosino, A. Rubino, Synth. Met. 2012, 161, 2714.

[113] A. Elschner, S. Kirchmeyer, W. Lövenich, U. Merker, K. Reuter, PEDOT: Principles and Applications of an Intrinsically Conductive Polymer, CRC Press: Taylor and Francis Group, Boca Raton, FL 2011, p. 131

[114] X. F. Peng, X. Y. Wu, X. X. Ji, J. Ren, Q. Wang, G. Q. Li, X. H. Yang, J. Phys. Chem. Lett. 2017, 8, 4691

[115] N. K. Kumawat, D. Gupta, D. Kabra, Energy Technol. 2017, 5, 1734.

[116] D. Abbaszadeh, G. A. H. Wetzelaer, H. T. Nicolai, P. W. M. Blom, J. Appl. Phys. 2014, 116, 224508.

[117] K. H. Yeoh, N. A. Talik, T. J. Whitcher, C. Y. B. Ng, K. L. Woon, J. Phys. D: Appl. Phys. 2014, 47, 205103.

[118] Y. Xu, Y. Wang, J. Liang, Y. Huang, Y. Ma, X. Wan, Y. Chen, Nano Res. 2009, 2, 343.

[119] J. H. Lee, J. J. Kim, J. Inf. Disp. 2013, 14, 39.

[120] C. Chang, S. Yang, Mater. Res. Express 2019, 6, 105304.

[121] M. Lai, A. Obliger, D. Lu, C. S. Kley, C. G. Bischak, Q. Kong, T. Lei, L. Dou, N. S. Ginsberg, D. T. Limmer, P. Yang, Proc. Natl. Acad. Sci. USA 2018, 115, 11929.

[122] C. G. Bischak, C. L. Hetherington, H. Wu, S. Aloni, D. F. Ogletree, D. T. Limmer, N. S. Ginsberg, Nano Lett. 2017, 17, 1028.

[123] Z. Xiao, Y. Yuan, Y. Shao, Q. Wang, Q. Dong, C. Bi, P. Sharma, A. Gruverman, J. Huang, Nat. Mater. 2015, 14, 193.

[124] X. Shan, J. Li, M. Chen, T. Geske, S. G. R. Bade, Z. Yu, J. Phys. Chem. Lett. 2017, 8, 2412.

[125] C. Eames, J. M. Frost, P. R. F. Barnes, B. C. O'Regan, A. Walsh, M. S. Islam, Nat. Commun. 2015, 6, 7497.

[126] H. Wang, M. Zhou, H. Luo, ACS Omega 2018, 3, 1445.

[127] S. Kim, S. Bae, S. Lee, K. Cho, K. D. Lee, H. Kim, S. Park, G. Kwon, S. Ahn, H. Lee, Y. Kang, H. Lee, D. Kim, Sci. Rep. 2017, 7, 1200.

[128] B. Rivkin, P. Fassl, Q. Sun, A. D. Taylor, Z. Chen, Y. Vaynzof, ACS Omega 2018, 3, 10042.

[129] F. A. Roghabadi, N. M. Rezaei Fumani, M. Alidaei, V. Ahmadi, S. M. Sadrameli, Sci. Rep. 2019, 9, 9448.

[130] Z. Huang, A. H. Proppe, H. Tan, M. I. Saidaminov, F. Tan, A. Mei, C. Tan, M. Wei, Y. Hou, H. Han, S. O. Kelley, E. H. Sargent, ACS Energy Lett. 2019, 4, 1521

[131] P. Gao, A. Yusoff, M. K. Nazeeruddin, Nat. Commun. 2018, 9, 5028.

[132] Y. Han, S. Park, C. Kim, M. Lee, I. Hwang, Nanoscale 2019, 11, 3546.

[133] S. Hou, M. K. Gangishetty, Q. Quan, D. N. Congreve, Joule 2018, 2, 2421.

[134] H. P. Kim, J. Kim, B. S. Kim, H. M. Kim, J. Kim, A. Rashid bin Mohd Yusoff, J. Jang, M. K. Nazeeruddin, Adv. Opt. Mater. 2017, 5, 1600920.

[135] M. K. Gangishetty, S. Hou, Q. Quan, D. N. Congreve, Adv. Mater. 2018, 30, 1706226

[136] N. K. Kumawat, A. Dey, A. Kumar, S. P. Gopinathan, K. L. Narasimhan, D. Kabra, ACS Appl. Mater. Interfaces 2015, 7, 13119.

[137] N. K. Kumawat, A. Dey, K. L. Narasimhan, D. Kabra, ACS Photonics 2015, 2, 349.

[138] H. Shao, X. Bai, G. Pan, H. Cui, J. Zhu, Y. Zhai, J. Liu, B. Dong, L. Xu, H. Song, Nanotechnology 2018, 29, 285706.
[139] H. Fang, W. Deng, X. Zhang, X. Xu, M. Zhang, J. Jie, X. Zhang, Nano Res. 2019, 12, 171

[140] M. Yuan, L. Quan, R. Comin, G. Walters, R. Sabatini, O. Voznyy, S. Hoogland, Y. Zhao, E. M. Beauregard, P. Kanjanaboos, Z. Lu, D. Kim, E. H. Sargent, Nat. Nanotechnol. 2016, 11, 872.

[141] O. Erdem, M. Olutas, B. Guzelturk, Y. Kelestemur, H. V. Demir, J. Phys. Chem. Lett. 2016, 7, 548.

[142] J. Jagielski, S. Kumar, M. Wang, D. Scullion, R. Lawrence, Y. T. Li, S. Yakunin, T. Tian, M. V. Kovalenko, Y. C. Chiu, E. J. G. Santos, S. Lin, C. J. Shih, Sci. Adv. 2017, 3, eaaq0208.

[143] H. Kim, L. Zhao, J. S. Price, A. J. Grede, K. Roh, A. N. Brigeman, M. Lopez, B. P. Rand, N. C. Giebink, Nat. Commun. 2018, 9, 4893.

[144] S. Nakahara, H. Tahara, G. Yumoto, T. Kawawaki, M. Saruyama, R. Sato, T. Teranishi, Y. Kanemitsu, J. Phys. Chem. C 2018, 122, 22188

[145] I. I. Fishchuk, A. Kadashchuk, M. Ullah, H. Sitter, A. Pivrikas J. Genoe, H. Bässler, Phys. Rev. B 2012, 86, 045207.

[146] M. Bouhassoune, S. L. M. van Mensfoort, P. A. Bobbert, R. Coehoorn, Org. Electron. 2009, 10, 437.

[147] H. C. Yoon, H. Lee, H. Kang, J. H. Oh, Y. R. Do, J. Mater. Chem. C 2018, 6, 13023

[148] Y. H. Song, S. H. Choi, J. S. Yoo, B. K. Kang, E. K. Ji, H. S. Jung, D. H. Yoon, Chem. Eng. J. 2017, 313, 461.

[149] J. Zhou, F. Huang, H. Lin, Z. Lin, J. Xu, Y. Wang, J. Mater. Chem. C 2016, 4, 7601

[150] Q. Zhang, Y. Yin, ACS Cent. Sci. 2018, 4, 668.

[151] Z. He, C. Zhang, Y. Dong, S.-T. Wu, Crystals 2019, 9, 59.

[152] C. Wang, Y. Su, T. Shih, G. Chen, Y. Chen, C. Lu, I. Yu, Z. Yang, H. Su, J. Mater. Chem. C 2018, 6, 12808.

[153] C. Sun, Y. Zhang, C. Ruan, C. Yin, X. Wang, Y. Wang, W. W. Yu, Adv. Mater. 2016, 28, 10088.

[154] J. Zhou, Z. Hu, L. Zhang, Y. Zhu, J. Alloys Compd. 2017, 708, 517.

[155] H. Wang, S. Lin, A. Tang, B. P. Singh, H. Tong, C. Chen, Y. Lee, T. Tsai, R. Liu, Angew. Chem., Int. Ed. 2016, 55, 7924.

[156] Q. Zhou, Z. Bai, W. G. Lu, Y. Wang, B. Zou, H. Zhong, Adv. Mater. 2016, 28, 9163

[157] Y. W. Zhang, G. Wu, H. Dang, K. Ma, S. Chen, Ind. Eng. Chem. Res. 2017, 56, 10053.

[158] F. Palazon, F. Di Stasio, Q. A. Akkerman, R. Krahne, M. Prato, L. Manna, Chem. Mater. 2016, 28, 2902.

[159] Z. Li, L. Kong, S. Huang, L. Li, Angew. Chem., Int. Ed. 2017, 56, 8134

[160] J. Zhang, Y. Yang, H. Deng, U. Farooq, X. Yang, J. Khan, J. Tang, H. Song, ACS Nano 2017, 11, 9294.

[161] X. Di, J. Jiang, Z. Hu, L. Zhou, P. Li, S. Liu, W. Xiang, X. Liang, Dyes Pigm. 2017, 146, 361.

[162] M. Leng, Z. Chen, Y. Yang, Z. Li, K. Zeng, K. Li, G. Niu, Y. He, Q. Zhou, J. Tang, Angew. Chem., Int. Ed. 2016, 55, 15012.

[163] Y. Wang, X. Li, V. Nalla, H. Zeng, H. Sun, Adv. Funct. Mater. 2017 , 27, 1605088

[164] T. Fan, J. Lü, Y. Chen, W. Yuan, J. Mater. Sci.: Mater. Electron. 2019, 30, 1084.

[165] H. Zhu, Y. Fu, F. Meng, X. Wu, Z. Gong, Q. Ding, M. V. Gustafsson, M. T. Trinh, S. Jin, X. Y. Zhu, Nat. Mater. 2015, 14, 636.

[166] J. Wang, P. Da, Z. Zhang, S. Luo, L. Liao, Z. Sun, X. Shen, S. Wu, G. Zheng, Z. Chen, Nanoscale 2018, 10, 10371

[167] S. W. Eaton, M. Lai, N. A. Gibson, A. B. Wong, L. Dou, J. Ma, L. W. Wang, S. R. Leone, P. Yang, Proc. Natl. Acad. Sci. USA 2016, 113, 1993.

[168] Y. Jia, R. A. Kerner, A. J. Grede, B. P. Rand, N. C. Giebink, Nat Photonics 2017, 11, 784.

[169] Y. Wang, X. Li, X. Zhao, L. Xiao, H. Zeng, H. Sun, Nano Lett. 2016, 16,448 .

[170] L. Wang, L. Meng, L. Chen, S. Huang, X. Wu, G. Dai, L. Deng, J. Han, B. Zou, C. Zhang, H. Zhong, J. Phys. Chem. Lett. 2019, 10, 3248. 
[171] C. Zhang, J. F. S. Fernando, K. L. Firestein, J. E. von Treifeldt, D. Siriwardena, X. Fang, D. Golberg, APL Mater. 2019, 7, 071110.

[172] K. Park, J. W. Lee, J. D. Kim, N. S. Han, D. M. Jang, S. Jeong, J. Park, J. K. Song, J. Phys. Chem. Lett. 2016, 7, 3703.

[173] T. Byrnes, N. Y. Kim, Y. Yamamoto, Nat. Phys. 2014, 10, 803.

[174] S. Kéna-Cohen, S. R. Forrest, Nat. Photonics 2010, 4, 371.

[175] P. Bhattacharya, B. Xiao, A. Das, S. Bhowmick, J. Heo, Phys. Rev. Lett. 2013, 110, 206403.

[176] C. Schneider, A. Rahimi-Iman, N. Y. Kim, J. Fischer, I. G. Savenko, M. Amthor, M. Lermer, A. Wolf, L. Worschech, V. D. Kulakovskii, I. A. Shelykh, M. Kamp, S. Reitzenstein, A. Forchel, Y. Yamamoto, S. Höfling, Nature 2013, 497, 348.

[177] D. W. Snoke, J. Keeling, Phys. Today 2017, 70, 54.

[178] W. Du, S. Zhang, Q. Zhang, X. Liu, Adv. Mater. 2018, 69, 1804894.

[179] R. Su, J. Wang, J. Zhao, I Xing, W. Zhao, C. Diederichs, T. C. H. Liew, Q. Xiong, Sci. Adv. 2018, 4, eaau0244.

[180] R. Su, S. Ghosh, S. Liu, C. Diederichs, T. C. H. Liew, Q. Xiong, Sci. Adv. 2018, 4, eaau0244.
[181] M. D. Birowosuto, D. Cortecchia, W. Drozdowski, K. Brylew, W. Lachmanski, A. Bruno, C. Soci, Sci. Rep. 2016, 6, 37254.

[182] H. Wei, J. Huang, Nat. Commun. 2019, 10, 1066.

[183] M. Nikl, A. Yoshikawa, Adv. Opt. Mater. 2015, 3, 463.

[184] Y. He, L. Matei, H. J. Jung, K. M. McCall, M. Chen, C. C. Stoumpos, Z. Liu, J. A. Peters, D. Y. Chung, B. W. Wessels, M. R. Wasielewski, V. P. Dravid, A. Burger, M. G. Kanatzidis, Nat. Commun. 2018, 9, 1609.

[185] A. J. Shields, Nat. Photonics 2007, 1, 215.

[186] B. Lounis, M. Orrit, Rep. Prog. Phys. 2005, 68, 1129.

[187] X. Lin, X. Dai, C. Pu, Y. Deng, Y. Niu, L. Tong, W. Fang, Y. Jin, X. Peng, Nat. Commun. 2017, 8, 1132.

[188] I. Kagomiya, K. Jimbo, K. Kakimoto, M. Nakayamaa, O. Massonb, Phys. Chem. Chem. Phys. 2014, 16, 10875.

[189] W. Lee, H. Li, A. B. Wong, D. Zhang, M. Lai, Y. Yu, Q. Kong, E. Lin, J. J. Urban, J. C. Grossman, P. Yang, Proc. Natl. Acad. Sci. USA 2017, 114, 8693.

[190] C. Ge, M. Hu, P. Wu, Q. Tan, Z. Chen, Y. Wang, J. Shi, J. Feng, J. Phys. Chem. C 2018, 122, 15973. 